\title{
Sensitivity Evaluation of Spectral Nudging Schemes in Historical Dynamical Downscaling for South Asia
}

\author{
Mehwish Ramzan, ${ }^{1,2}$ Suryun Ham, ${ }^{3}$ Muhammad Amjad, ${ }^{4}$ \\ Eun-Chul Chang, ${ }^{5}$ and Kei Yoshimura ${ }^{1,2}$ \\ ${ }^{1}$ Atmosphere and Ocean Research Institute, The University of Tokyo, Kashiwa, Chiba, Japan \\ ${ }^{2}$ Institute of Industrial Science, The University of Tokyo, Meguro, Tokyo, Japan \\ ${ }^{3}$ Climate Prediction Department, APEC Climate Center, Busan, Republic of Korea \\ ${ }^{4}$ Global Change Impact Studies Centre, Islamabad, Pakistan \\ ${ }^{5}$ Department of Atmospheric Science, Kongju National University, Gongju, Republic of Korea
}

Correspondence should be addressed to Mehwish Ramzan; mehwish.ramzan@gmail.com

Received 19 October 2016; Revised 10 January 2017; Accepted 27 February 2017; Published 27 April 2017

Academic Editor: Charles Jones

Copyright (C) 2017 Mehwish Ramzan et al. This is an open access article distributed under the Creative Commons Attribution License, which permits unrestricted use, distribution, and reproduction in any medium, provided the original work is properly cited.

Sensitivity experiments testing two scale-selective bias correction (SSBC) methods have been carried out to identify an optimal spectral nudging scheme for historical dynamically downscaled simulations of South Asia, using the coordinated regional climate downscaling experiment (CORDEX) protocol and the regional spectral model (RSM). Two time periods were selected under the category of short-term extreme summer and long-term decadal analysis. The new SSBC version applied nudging to full wind components, with an increased relaxation time in the lower model layers, incorporating a vertical weighted damping coefficient. An evaluation of the extraordinary weather conditions experienced in South Asia in the summer of 2005 confirmed the advantages of the new SSBC when modeling monsoon precipitation. Furthermore, the new SSBC scheme was found to predict precipitation and wind patterns more accurately than the older version in decadal analysis, which applies nudging only to the rotational wind field, with a constant strength at all heights.

\section{Introduction}

South Asia has a diverse climate, the world's highest mountains, and a well-developed monsoon system. Like many other parts of the world, South Asia is increasingly experiencing abnormal climate extremes. "Turn Down the Heat," a report launched by the World Bank [1], warned that although all nations will suffer from the impact of climate change, the most vulnerable among them would be in subSaharan Africa, South Asia, and Southeast Asia. These climate extremes include heavy precipitation, heat waves, tropical cyclones, and droughts. "Stories of Impact," published by the International Finance Corporation (IFC) of the World Bank Group for South Asia (2014), brought attention to the melting glaciers of the Himalayas which pose a great risk of flood outbursts and an increase in sea level. Therefore, coastal countries such as Bangladesh and The Maldives are at great risk. Abnormal monsoon rainfall is also predicted in South Asia (http://www.ifc.org/). These recent reports emphasize the increasing frequency of extreme events in South Asia and encourage further study of this region.

Climate models are designed to deliver hindcast, the study of climate variability, and projections of future climate and to study the impacts of anthropogenic influences. General circulation models (GCMs) with high spatial resolution require large quantities of computational resources and time. Alternatively, high-resolution regional climate models (RCMs) facilitate more rapid and less costly localized climate studies compared to GCMs.

In this work, the regional spectral model (RSM) originally developed at the National Center for Environmental Predictions (NCEP) by Juang and Kanamitsu [2] is 
applied to the South Asian region with a high resolution. The outcome of this research will contribute to the world climate research program (WCRP), which generates the high-resolution regional information for all land parts of the world. The project under which these efforts are being conducted is known as the coordinated regional climate downscaling experiment (CORDEX). A large community is required to participate in this effort of generating future climate projections for multiple regions of the world (http://wcrp-cordex.ipsl.jussieu.fr). A $50 \mathrm{~km}$ resolution is selected for the first phase of this project. This CORDEXSouth Asian experiment (CORDEX-SA) using RSM is initiated to identify the optimal spectral nudging scheme for reproducing the summer and winter precipitation of South Asia.

Previous studies demonstrated prevalent issues in climate modeling of the South Asian region. According to Sabin et al. [3], the South Asian monsoon (SAM) is heterogeneous in both time and space. The findings of Gadgil and Sajani [4] showed the limited performance of coarse-resolution atmospheric GCMs for simulating SAM in the subcontinents. Their findings highlighted the wet precipitation bias over the Equatorial Indian Ocean (EIO), which they attributed to the low resolution of GCMs and inefficient model physical processes, such as moist convection, boundary layer flux, and radiative effects of GCMs.

The dynamic downscaling approach, which nests the RCM over GCM in the region of interest, sometimes creates a systematic bias in the newly formed regional model domains. Multiple methods are adopted to remove or minimize these biases. According to $\mathrm{Xu}$ and Yang [5], reanalysis-driven RCMs exhibit less bias than GCM-driven RCMs but their study is conducted in North America, which needs further analysis to assess their applicability in other regions of the world. In both cases, bias is an inevitable part of downscaling. Several feasibility studies have been conducted within the climate modeling community to mitigate these biases using spectral nudging techniques (e.g., [6-8]). Kanamaru and Kanamitsu [9], for example, minimized systematic RSM biases, through a combination of spectral tendency damping correction and areal averaging of temperature, humidity, and pressure. This scale-selective bias correction (SSBC) method may cause a number of problems to the lateral boundary relaxation, due to the effective reduction of largescale discontinuities near the boundary.

Kanamitsu et al. [10] refined the SSBC method by replacing spectral tendency nudging with field nudging, which removed the errors of large-scale interannual variability of the seasonal mean. This field nudging is applied only to the rotational component of the wind. This modified method reduces the model's synoptic and planetary scale errors. Their results showed improved accuracy for variables such as geopotential height, temperature, and wind at $500 \mathrm{hPa}$ and agreed with the global analysis. However, the variables such as surface precipitation and near-surface temperature did not show a notable improvement in this category. This method by Kanamitsu et al. [10] is present by default in RSM. It will be referred to as SSBC_Def here.
Hong and Chang [11] revised the RSM SSBC_Def method (SSBC_New). The SSBC_New method applied full wind nudging and a vertically weighted damping coefficient. Alternatively, SSBC_Def applies a damping coefficient that is independent of height, which uses a small coefficient value as the default. The SSBC_New results of Hong and Chang [11] showed wind fields similar to the driving global analysis over East Asia, but it caused distortion of temperature and geopotential height. SSBC_New gradually reduced the vorticity nudging from the top of the model to the ground, which improved precipitation simulation results. The vertically weighted damping coefficient applied in SSBC_New gave a damping time scale of less than $2 \mathrm{~h}$ at $800 \mathrm{hPa}$ and above, while it is greater than $6 \mathrm{~h}$ below $850 \mathrm{hPa}$ (see Hong and Chang [11] Figure 1, for a detailed description). Their 10-year sensitivity experiment simulation of June, July, and August (JJA) data for the CORDEX-East Asia domain further confirmed the superiority of SSBC_New over SSBC_Def in terms of capturing monsoon rainfall over East Asia.

The current study extends the application of previous SSBC schemes over the new domain of CORDEX-SA and focuses on the South Asian summer (JJA) and winter (DJF) monsoon seasons. The seasonal reversal of monsoon winds in South Asia is not fully understood, and therefore the winds and associated precipitation need to be studied in detail [1215]. These variables appear to act as controlling factors in shaping this regional rainfall phenomenon.

This study is based on downscaled global NCEP and Department of Energy (DOE) reanalysis-2 (R-2) data using RSM. The CORDEX-SA region covers a large area starting from the Middle East, East and South Africa, parts of Central Asia, all of South Asia, and parts of East and Southeast Asia. These regions lie within tropical and subtropical zones where, according to Hong and Chang [11], the ageostrophic wind is more significant. It is therefore more sensitive to vertical weighted damping, which reduces the strength of nudging at lower atmospheric levels. SSBC_Def and SSBC_New are evaluated by considering both short- and long-term simulations including (i) an extraordinary summer (2005), (ii) 10 years of summer and winter simulations (1981-1990), and (iii) very heavy precipitation analysis of 1981-1990. The reason of selecting this time period (1981-1990) is to make it coincide with our 20th century experiment of CORDEX-SA (1980-2005) for which these sensitivity tests are conducted. Also, this time period will express the model's capability of performing in data sparse decade. This can be used as a baseline for the study of hindcast of this region.

The structure of this paper is as follows. Section 2 presents the experimental design, a description of SSBC methods, and the observation/reanalysis datasets used. Section 3 discusses the results obtained for both short- and long-term simulations with SSBC_Def and SSBC_New. Section 4 will present the conclusions drawn from this work.

\section{Experimental Setup}

2.1. Model and Datasets Used. NCEP RSM, which is a primitive equation model comprising momentum, hydrostatic, thermodynamic, and mass continuity equations [9], is used 


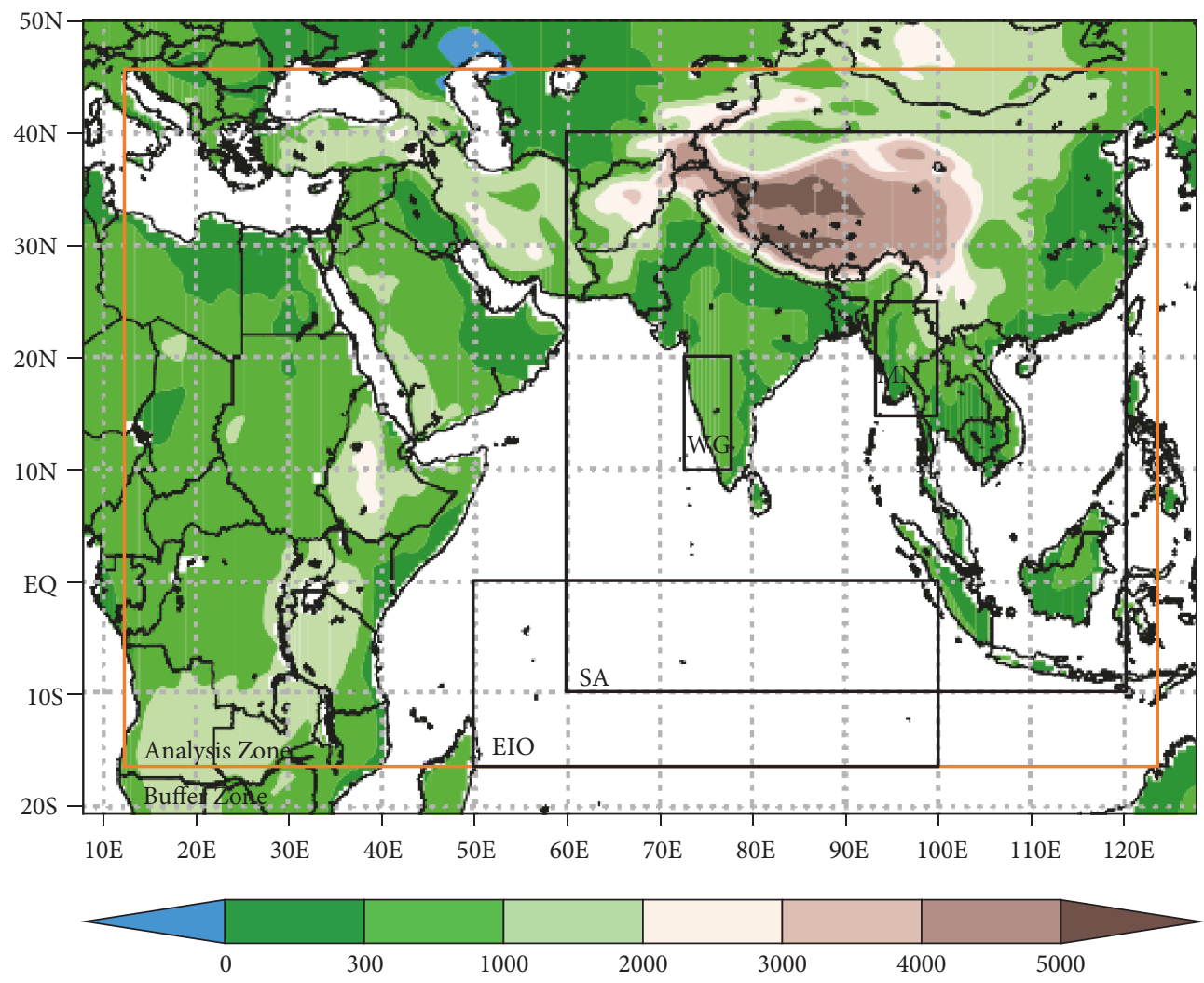

Figure 1: Regional model domain and orography $(\mathrm{m})$ of the downscaling experiment for CORDEX-South Asia. The analysis zone is used throughout the experiment (indicated by an orange box) excluding the buffer zone (five grid columns from each side). Black boxes indicate the subdomains selected for study. The coordinates of analysis zone and subdomains are given in Table 1.

in this research. The major physical process of RSM includes the simplified Arakawa Schubert (SAS) convection scheme [16], the NOAH land surface model [17], the Chou scheme for long- and short-wave radiation [18], the planetary boundary scheme [19], gravity wave drag [20, 21], enhanced topography, and vertical or horizontal diffusion $[19,22]$. Figure 1 shows the downscaling experimental region for CORDEX$\mathrm{SA}$, which extends from $7^{\circ} \mathrm{E}$ to $128^{\circ} \mathrm{E}$ and $20^{\circ} \mathrm{S}$ to $50^{\circ} \mathrm{N}$. The horizontal grid spacing is $\sim 50 \mathrm{~km}$. The number of grid points for west-east and north-south are $257 \times 169$. RSM follows a terrain sigma coordinate system, which includes 28 levels. R2 of a $6 \mathrm{~h}$ dataset is used for the initial and lateral boundary conditions [23]. Five grid columns from the CORDEX-SA boundaries are deemed as buffer zone, while the main study is performed within the analysis zone.

The analysis is performed on major domain, that is, CORDEX-SA, and multiple subdomains. These subdomains are selected based on their importance in receiving the influence of South and East Asian summer monsoon from its onset, propagation, and demise (end). A detailed description of the study areas is given in Table 1, along with coordinates and abbreviations.

Tropical Rainfall Measuring Mission (TRMM) satellite monthly data 3B43 (ver. 7), with a spatial resolution of $0.25^{\circ} \times 0.25^{\circ}$, are used for the evaluation of JJA 2005 precipitation for both SSBC schemes (https://mirador.gsfc .nasa.gov/cgi-bin/mirador/presentNavigation.pl?tree=project
TABLE 1: Description of major domain (CORDEX-SA) and subdomains used.

\begin{tabular}{lccc}
\hline \multirow{2}{*}{ Domain } & \multirow{2}{*}{ Abbreviations } & \multicolumn{2}{c}{ Coordinates } \\
& & Longitude & Latitude \\
\hline CORDEX-South Asia & CORDEX-SA & $12^{\circ} \mathrm{E}-123^{\circ} \mathrm{E}$ & $15^{\circ} \mathrm{S}-45^{\circ} \mathrm{N}$ \\
South Asia & SA & $60^{\circ} \mathrm{E}-120^{\circ} \mathrm{E}$ & $10^{\circ} \mathrm{S}-40^{\circ} \mathrm{N}$ \\
Myanmar & $\mathrm{MN}$ & $92^{\circ} \mathrm{E}-100^{\circ} \mathrm{E}$ & $15^{\circ} \mathrm{N}-25^{\circ} \mathrm{N}$ \\
Equatorial Indian Ocean & $\mathrm{EIO}$ & $50^{\circ} \mathrm{E}-100^{\circ} \mathrm{E}$ & $15^{\circ} \mathrm{S}-0^{\circ}$ \\
Western Ghats & $\mathrm{WG}$ & $72^{\circ} \mathrm{E}-78^{\circ} \mathrm{E}$ & $10^{\circ} \mathrm{N}-20^{\circ} \mathrm{N}$ \\
\hline
\end{tabular}

\&project $=$ TRMM $\&$ dataGroup $=$ Gridded $\&$ dataset $=3 \mathrm{~B} 43: \% 20$ Monthly\%200.25\%20x\%200.25\%20degree\%20merged $\% 20$ TRMM\%20and\%20other\%20sources\%20estimates\&version $=006$ ). The JJA 2005 and 10-year surface precipitation analysis for JJA and DJF are validated using monthly precipitation data from the Climate Prediction Center (CPC) using merged analysis of precipitation (CMAP) (http://www.esrl.noaa.gov/psd/) with a spatial resolution of $2.5^{\circ} \times 2.5^{\circ}$. Two reanalysis datasets are used for the evaluation of $850 \mathrm{hPa}$ atmospheric variables such as zonal and meridional winds, relative humidity, air temperature, and geopotential height. These datasets are (i) European Reanalysis (ERA-interim) monthly data (http://apps.ecmwf .int/datasets/data/interim-full-moda/levtype $=$ sfc/) with a 
TABLE 2: List of observation and reanalysis datasets used in validating the current study. The known biases are mentioned along with the temporal and spatial resolutions.

\begin{tabular}{|c|c|c|c|c|c|c|}
\hline $\begin{array}{l}\text { S. } \\
\text { number }\end{array}$ & Dataset & Version & $\begin{array}{l}\text { Temporal } \\
\text { resolution }\end{array}$ & $\begin{array}{c}\text { Spatial } \\
\text { resolution }\end{array}$ & Coverage & Known biases \\
\hline 1 & TRMM & 3B43_V7 & Monthly & $0.25^{\circ} \times 0.25^{\circ}$ & Land + ocean & $\begin{array}{l}\text { Underestimation of precipitation over intense } \\
\text { convection areas and at high latitudes (see details } \\
\text { at https://climatedataguide.ucar.edu/climate- } \\
\text { data/trmm-tropical-rainfall-measuring-mission) }\end{array}$ \\
\hline 2 & NCEP-CFSR & 93.2 & $\begin{array}{l}\text { 6-hour diurnal } \\
\text { monthly means }\end{array}$ & $0.5^{\circ} \times 0.5^{\circ}$ & Land + ocean & $\begin{array}{c}\text { Less tested interaction between ocean and } \\
\text { atmosphere used indirectly and uncoupled } \\
\text { reanalysis (see details at } \\
\text { https://climatedataguide.ucar.edu/climate-data/ } \\
\text { climate-forecast-system-reanalysis-cfsr) }\end{array}$ \\
\hline 3 & APHRODITE & V1101 & Daily & $0.5^{\circ} \times 0.5^{\circ}$ & Land & $\begin{array}{c}\text { Heterogeneity in station locations, gauges, time, } \\
\text { and seasons (see details at } \\
\text { https://climatedataguide.ucar.edu/climate- } \\
\text { data/aphrodite) }\end{array}$ \\
\hline 4 & ERA-interim & $* * *$ & Monthly & $\begin{array}{l}\text { T255 spectral } \\
\text { (approx. } \\
80 \mathrm{~km})\end{array}$ & Land + ocean & $\begin{array}{c}\text { Extreme precipitation and evaporation over the } \\
\text { oceans, positive temperature and humidity bias } \\
\text { below } 850 \mathrm{hPa} \text {, and poor capability in } \\
\text { representing low-level inversion (see details at } \\
\text { https://climatedataguide.ucar.edu/climate-data/ } \\
\text { era-interim) }\end{array}$ \\
\hline 5 & CMAP & $* * *$ & Monthly & $2.5^{\circ} \times 2.5^{\circ}$ & Land + ocean & $\begin{array}{c}\text { Dependency on quality of input data, more } \\
\text { ambiguity towards higher latitudes especially at } \\
\text { polar regions (see details at } \\
\text { https://climatedataguide.ucar.edu/climate- } \\
\text { data/cmap-cpc-merged-analysis-precipitation) }\end{array}$ \\
\hline 6 & Reanalysis-2 & $\begin{array}{l}\text { NCEP- } \\
\text { DOE }\end{array}$ & Monthly & $2.5^{\circ} \times 2.5^{\circ}$ & Land + ocean & $\begin{array}{l}\text { First generation reanalysis data with less moisture } \\
\text { variability in time and space over the oceans (see } \\
\text { details at } \\
\text { https://climatedataguide.ucar.edu/climate-data/ } \\
\text { ncep-reanalysis-r2) }\end{array}$ \\
\hline
\end{tabular}

TRMM: Tropical Rainfall Measuring Mission.

NCEP: National Centers for Environmental Prediction.

CFSR: Climate Forecast System Reanalysis.

ERA: European Centre for Medium-Range Weather Forecasts.

CMAP: Climate Prediction Centre (CPC) merged analysis of precipitation.

DOE: Department of Energy.

$* * *$ means that the versions of these datasets are not available.

spatial resolution of $\sim 80 \mathrm{~km}$ (T255 spectral) and (ii) NCEP Climate Forecast System Reanalysis (CFSR) 093.2 as a 6-h diurnal and regular monthly mean with $0.5^{\circ}$ horizontal resolution [24] (similar resolution as this current experiment).

Daily surface precipitation $\left(\mathrm{mm} \mathrm{day}^{-1}\right)$ from gauge based product of Asian Precipitation-Highly-Resolved Observational Data Integration Towards Evaluation of the Water Resources (ver. 1101; APHRO) [25] is used for the 10-year very heavy precipitation analysis. The precipitation events are defined as very heavy when they fall in the upper $1 \%$ of all the precipitation events. Therefore, the percentile values are used to calculate them. Due to the heterogeneity between APHRO and CMAP in terms of land and ocean coverage, we divide the analysis into two categories, that is, (a) land plus ocean relative to CMAP and (b) land only relative to APHRO. The comparison for 99th percentile precipitations is made on the basis of regional averages. The regional description is given in Table 1.

It is important to mention that multiple datasets are used in this study based upon their spatial and temporal resolution and areal coverage. A detailed description of all the observation and reanalysis datasets is given in Table 2. We also listed the known biases that exist in these datasets.

2.2. Scale-Selective Bias Correction Experiment. Both SSBC Def and SSBC_New methods of RSM are applied to the CORDEX-SA region. The short-term simulation is considered as a special case because of the unique heavy rainfall event that occurred in Maharashtra, Mumbai, India, on July 26, 2005, which caused considerable damage in the metropolitan area. The entire summer of 2005 is thus selected to run the SSBC_Def and SSBC_New to assess the performance of both correction methods. A 10-year analysis 
TABLE 3

(a) Comparison of the root-mean-square error (RMSE) and spatial correlation (SCORR) of SSBC_Def and SSBC_New, compared with TRMM_3B43V7 monthly surface precipitation data $\left(\mathrm{mm} \mathrm{d}^{-1}\right)$ for June-JulyAugust (JJA), 2005 (the bold parts represent the best correlation and least error)

\begin{tabular}{lcccc}
\hline \multirow{2}{*}{ Domain } & \multicolumn{2}{c}{ SSBC_Def } & \multicolumn{2}{c}{ SSBC_New } \\
& RMSE & SCORR & RMSE & SCORR \\
\hline CORDEX-SA & 3.730 & 0.630 & $\mathbf{3 . 1 7 0}$ & $\mathbf{0 . 7 4 0}$ \\
SA & 4.690 & 0.530 & $\mathbf{4 . 2 1 0}$ & $\mathbf{0 . 6 4 0}$ \\
MN & $\mathbf{5 . 2 1 0}$ & $\mathbf{0 . 8 1 0}$ & 7.610 & 0.650 \\
EIO & 8.010 & -0.100 & $\mathbf{3 . 9 7 0}$ & $\mathbf{0 . 3 2 0}$ \\
WG & 7.360 & 0.700 & $\mathbf{5 . 7 9 0}$ & $\mathbf{0 . 8 2 0}$ \\
\hline
\end{tabular}

(b) The same as (a) but with CMAP observations for JJA, 2005

\begin{tabular}{lcccc}
\hline \multirow{2}{*}{ Domain } & \multicolumn{2}{c}{ SSBC_Def } & \multicolumn{2}{c}{ SSBC_New } \\
& RMSE & SCORR & RMSE & SCORR \\
\hline CORDEX-SA & 3.890 & 0.573 & $\mathbf{3 . 1 9 1}$ & $\mathbf{0 . 7 1 9}$ \\
SA & 5.005 & 0.415 & $\mathbf{4 . 2 6 6}$ & $\mathbf{0 . 5 8 0}$ \\
MN & $\mathbf{1 1 . 6 3 8}$ & $\mathbf{0 . 2 1 3}$ & 13.128 & 0.134 \\
EIO & 7.832 & -0.099 & $\mathbf{3 . 7 8 2}$ & $\mathbf{0 . 4 2 1}$ \\
WG & 7.174 & $\mathbf{0 . 8 8 7}$ & $\mathbf{5 . 3 4 5}$ & 0.855 \\
\hline
\end{tabular}

(1981-1990) is performed, with 1980 as a spin-up year, to assess both SSBC methods.

\section{Results and Discussion}

3.1. Extreme Summer, 2005. The results for SSBC_Def and SSBC_New for JJA, 2005, precipitation are presented in Table 3(a) and Figure 2, respectively. The R-2 dataset for surface precipitation is included for comparison, to assess how downscaling techniques add value over the coarse-resolution global reanalysis data. CMAP surface precipitation is also included to be consistent with the multiyear downscaling.

The results of JJA, 2005, for CORDEX-SA show a higher spatial correlation (SCORR) (0.74) with the lowest rootmean-square error (RMSE; $3.17 \mathrm{~mm} \mathrm{~d}^{-1}$ ) for SSBC_New when compared with SSBC_Def and R-2. R-2 gave an RMSE of $3.61 \mathrm{~mm} \mathrm{~d}^{-1}$ with 0.66 SCORR (values not given in Table 3 for R-2) whereas SSBC_Def gave a higher RMSE (3.73 $\left.\mathrm{mm} \mathrm{d}^{-1}\right)$ and lower SCORR (0.63; the lowest scores). By comparing the statistical results of both SSBCs with R-2, it can be seen that the downscaling technique enhanced RSM's ability in capturing surface precipitation when SSBC_New is used. However, it suppressed the model's ability when SSBC_Def is selected.

The most obvious feature of JJA, 2005, is the overestimated precipitation simulated by R-2 (Figure 2(c)) and SSBC_Def (Figure 2(e)) over the western EIO. The results of SSBC_New, however, show a closer simulation of the observed precipitation (see Figures 2(a), 2(b), and 2(f) and Table 3(a) for EIO scores). Another feature of this analysis is the spread of precipitation along the hills and mountains of South Asia [26-29]. For example, SSBC_Def reflects less accurately the observed precipitation along the Western Ghats (WG) of India and, specifically, over the Mumbai region versus SSBC_New. Because of the coarse resolution, R-2 data do not show fine alignment of precipitation along the WG of India, which suggests the importance of high resolution in mountainous regions.

Subdomain analysis for JJA, 2005 (Table 3(a)), shows that, regardless of the subdomain selected for surface precipitation analysis, the results for SSBC_New are better than those for SSBC_Def, except for Myanmar (MN).

The CMAP (see Table 3(b) and Figure 2(b)) comparison with both schemes for CORDEX-SA and its subdomains shows improved results for SSBC_New versus SSBC_Def similar to those listed in Table 3(a), with the only exception being the higher SCORR of SSBC_Def for WG.

Figure 2(d) shows the APHRO results for JJA, 2005, surface precipitation $\left(\mathrm{mm} \mathrm{d}^{-1}\right)$. The land part of the SSBC_New is more closer to APHRO than SSBC_Def specially over South China. More evaluation of both SSBCs can be interpreted from Figure 4 which is showing the spatial distribution of RMSE $\left(\mathrm{mm} \mathrm{d}^{-1}\right)$ for JJA, 2005. All observation datasets of Figure 2 are used to evaluate SSBC schemes. It is clear from Figures 3(a), 3(c), 3(b), and 3(d) that SSBC_Def is showing more errors in simulating ocean part of the CORDEX-SA domain.SSBC_New, on the other hand, is showing less errors not only over the ocean but also over the land (see Figures 3(e) and 3(f)). Thus, SSBC_New is the best option regardless of the spatial resolution of the observations for single season extreme summer analysis, that is, JJA, 2005.

The reason for the improved precipitation obtained with SSBC_New may be attributable to the vertically weighted damping coefficient and full wind nudging of SSBC_New. SSBC_Def has a limitation for synoptic-to-planetary scale precipitation, similar to the findings of Hong and Chang [11] for CORDEX-EA.

3.2. 10-Year Climatology Evaluation. The results of simulated precipitation climatology for JJA and DJF are presented in Figure 4, and their differences are presented in Figure 5. The CMAP precipitation for JJA climatology presented a typical SAM pattern, from west to east with heavy precipitation along the WG of India, Bay of Bengal, and the highlands of MN. Both SSBC experiments simulated JJA climatology, but with greater wet bias over the abovementioned regions (see Figures 5(a) and 5(b)). A prominent dry bias relative to CMAP is also observed over the northwest of India and the east of EIO. A comparison of both SSBC methods over the EIO shows that SSBC_New greatly reduced the dry bias of SSBC_ Def. Similarly, the wet bias over Southeastern China and dry bias over the South China Sea shown by SSBC_Def are minimized by SSBC_New. The RMSE and SCORR (see Table 4) further confirmed the improved performance of SSBC_New over SSBC_Def.

The winter climatology of CMAP (see Figure $4(\mathrm{~d})$ ) shows the precipitation band below the entire equatorial belt covering all the regions beneath it. Both SSBC methods captured this winter climatology (see Figures 4(e) and 4(f)). Dry precipitation bias over Southeast Asia is observed in both 


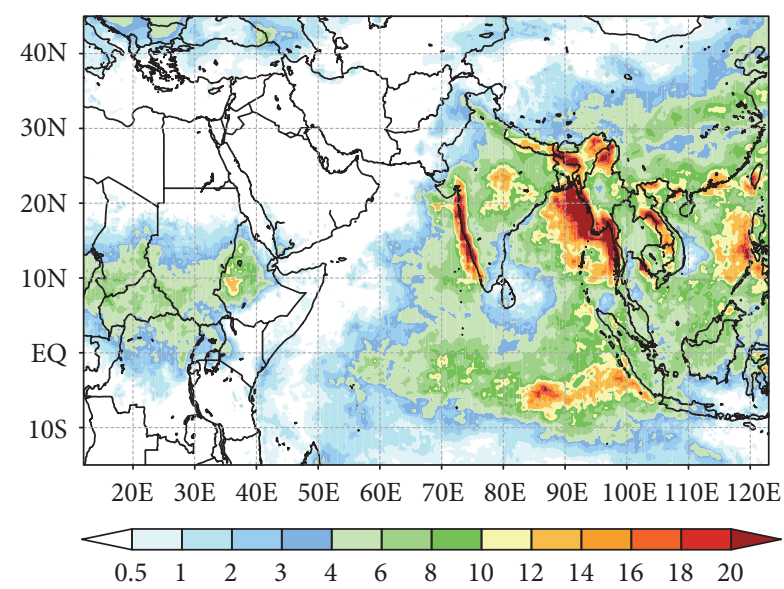

(a) TRMM 3B43_V7, JJA2005

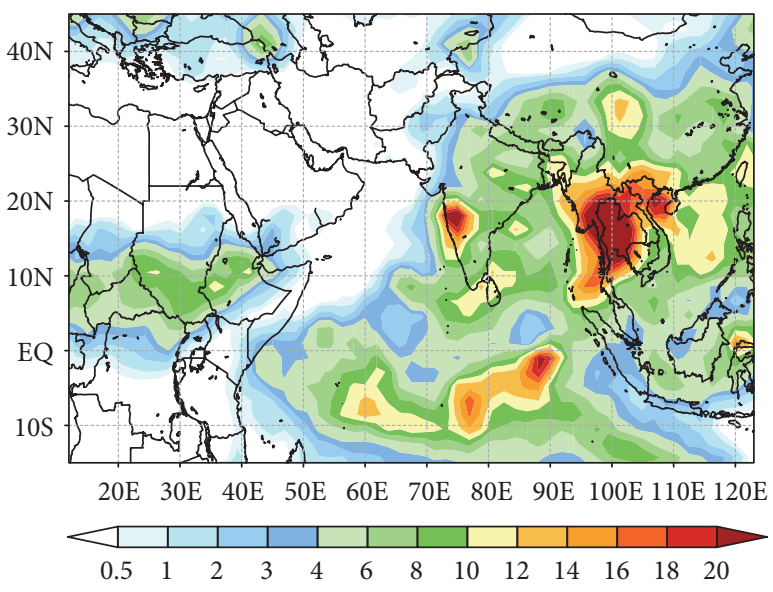

(c) Reanalysis-2, JJA2005

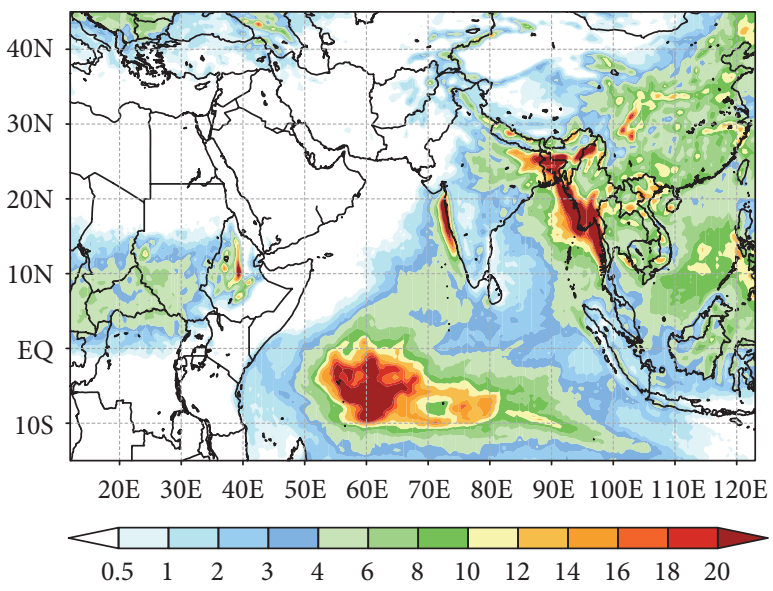

(e) SSBC_Def, JJA2005

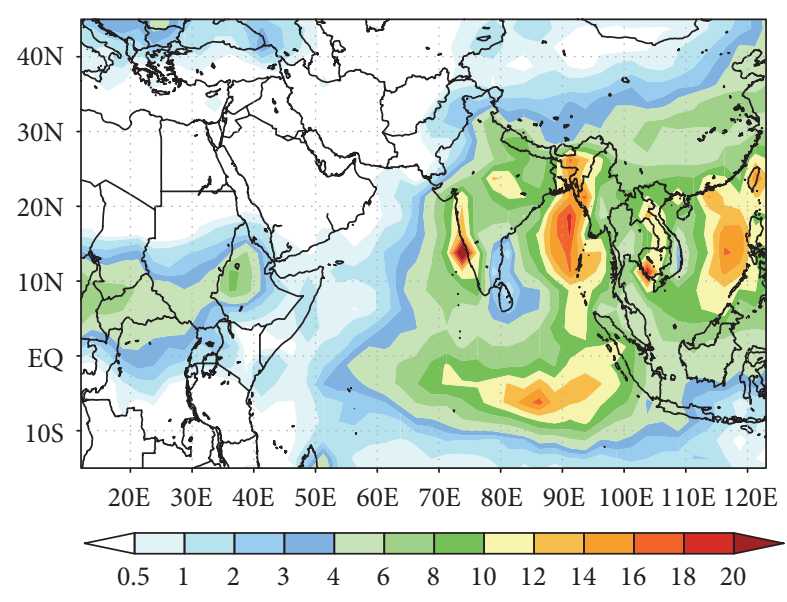

(b) CMAP, JJA2005

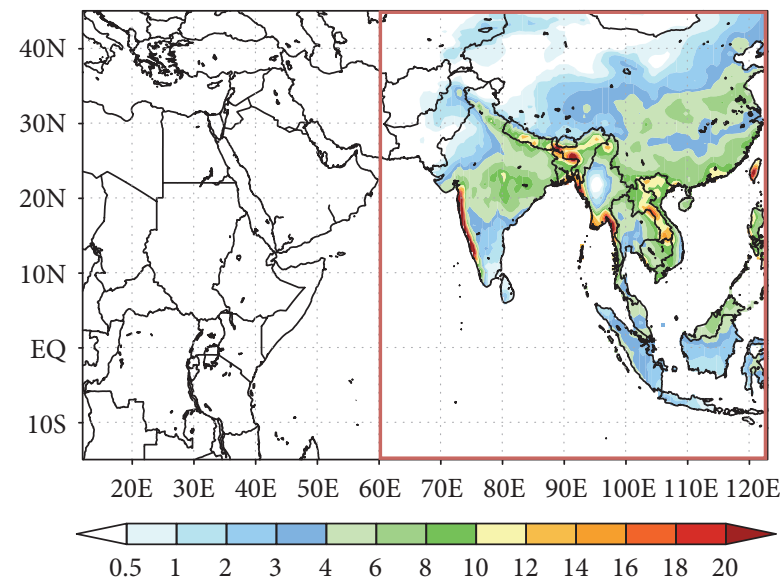

(d) APHRO, JJA2005

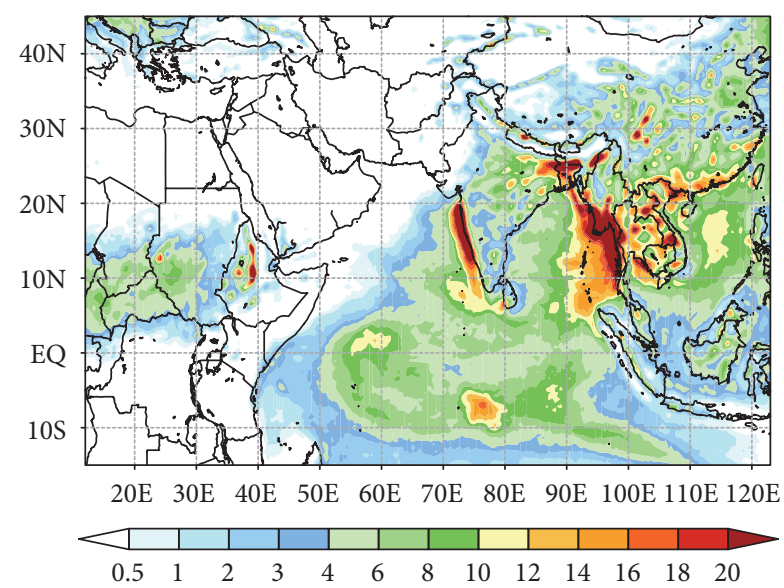

(f) SSBC_New, JJA2005

FIGURE 2: Comparison of SSBC_Def (e) and SSBC_New (f) surface precipitation $\left(\mathrm{mm} \mathrm{d}^{-1}\right)$ for JJA, 2005, with TRMM satellite 3B43_V7 (a), CMAP (b), Reanalysis-2 (c), and APHRODITE (d) surface precipitation $\left(\mathrm{mm} \mathrm{d}^{-1}\right)$. Red box in (d) indicates the spatial coverage by APHRODITE dataset. Further analysis will be conducted within this region.

schemes (see Figures 5(c) and 5(d)). Over the Indian Ocean, a prominent dry bias is observed in SSBC_Def, whereas SSBC_New shows a slight wet bias. The basic statistical score for DJF (see Table 4) shows a greater SCORR and lower
RMSE for SSBC_New than SSBC_Def. One reason for a dry bias over the oceans can be a systematic bias from the boundary conditions (see Table 2). R-2 is known for less moisture availability over the oceans, meaning that the 


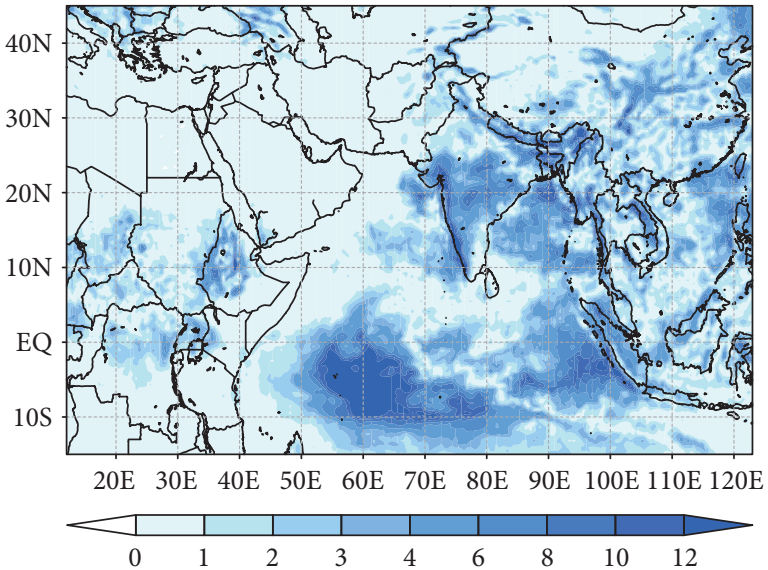

(a) SSBC_Def, TRMM, JJA

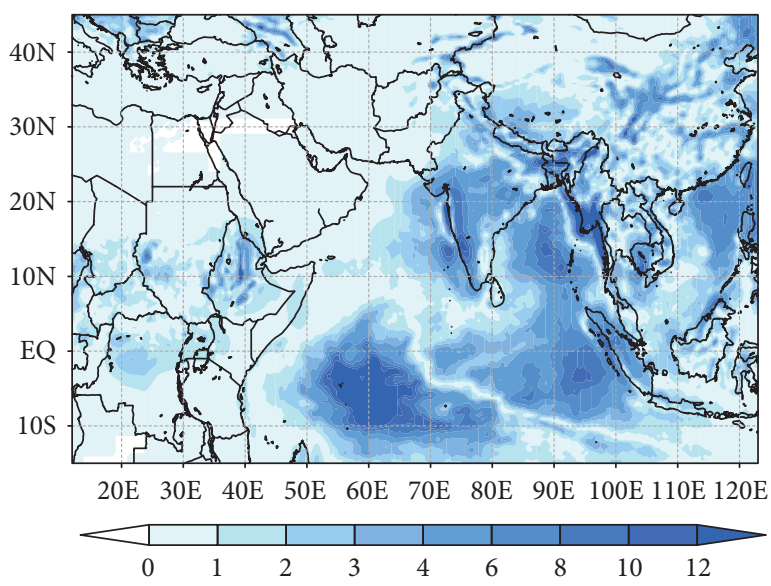

(c) SSBC_Def, CMAP, JJA

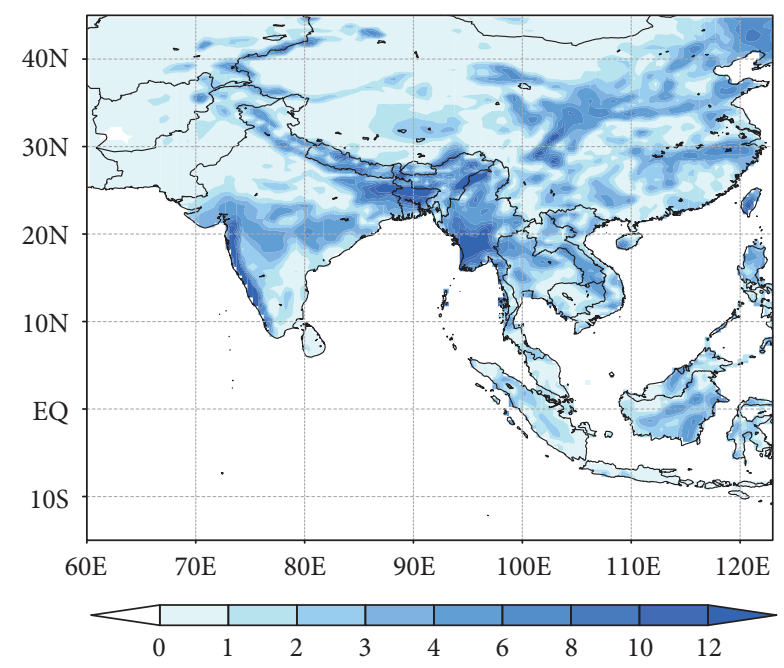

(e) SSBC_Def, APHRO, JJA

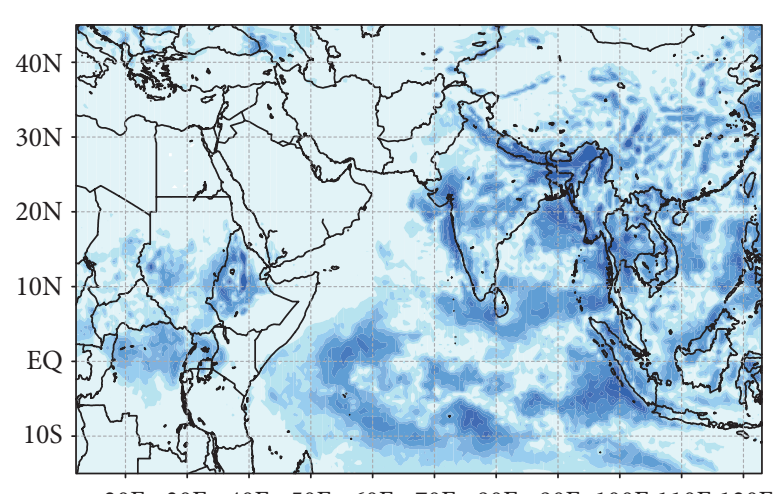

$20 \mathrm{E} \quad 30 \mathrm{E} \quad 40 \mathrm{E} \quad 50 \mathrm{E} \quad 60 \mathrm{E} \quad 70 \mathrm{E} \quad 80 \mathrm{E} \quad 90 \mathrm{E} \quad 100 \mathrm{E} 110 \mathrm{E} 120 \mathrm{E}$

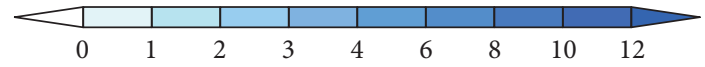

(b) SSBC_New, TRMM, JJA
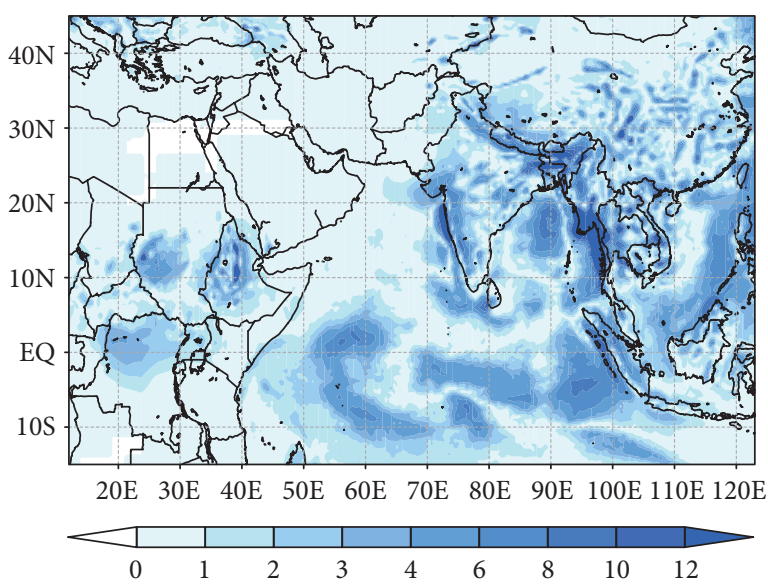

(d) SSBC_New, CMAP, JJA

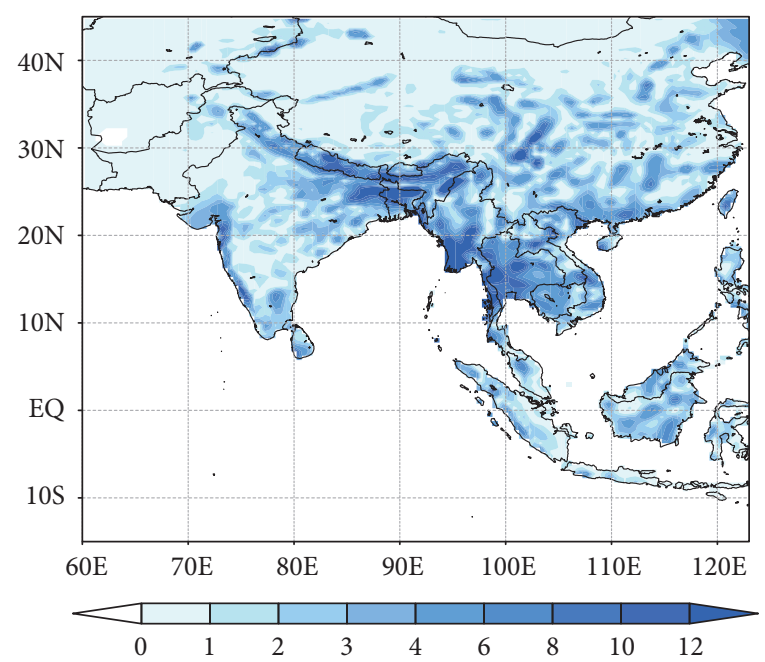

(f) SSBC_New, APHRO, JJA

FIGURE 3: Root-mean-square error (RMSE) comparison of surface precipitation for June-July-August (JJA), 2005, of SSBC_Def (left column) and SSBC_New (right column) relative to TRMM ( $\mathrm{a}$ and b), CMAP (c and d), and APHRODITE (e and f) surface precipitation ( $\mathrm{mm} \mathrm{d}^{-1}$ ). 


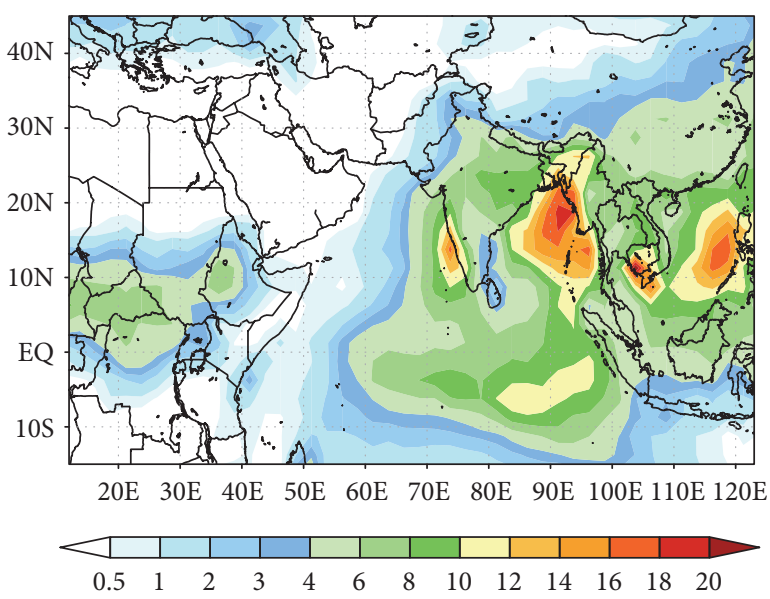

(a) CMAP, JJA

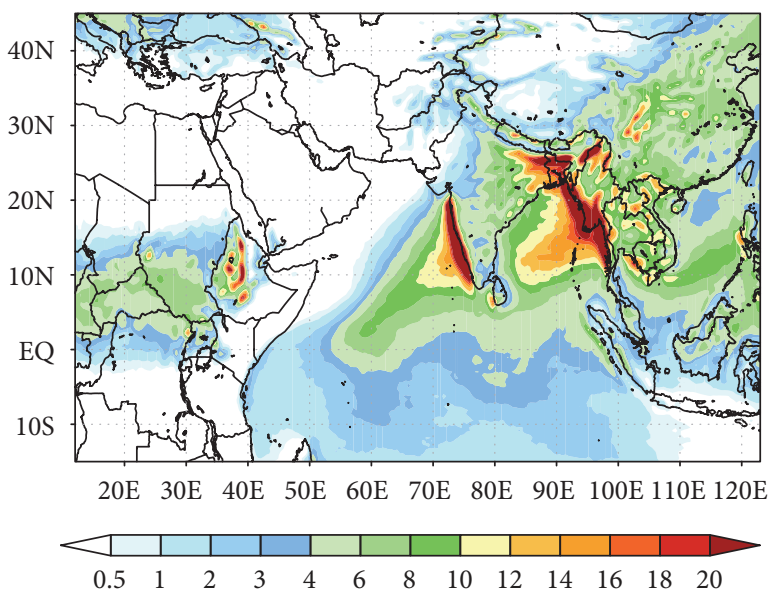

(b) SSBC_Def, JJA

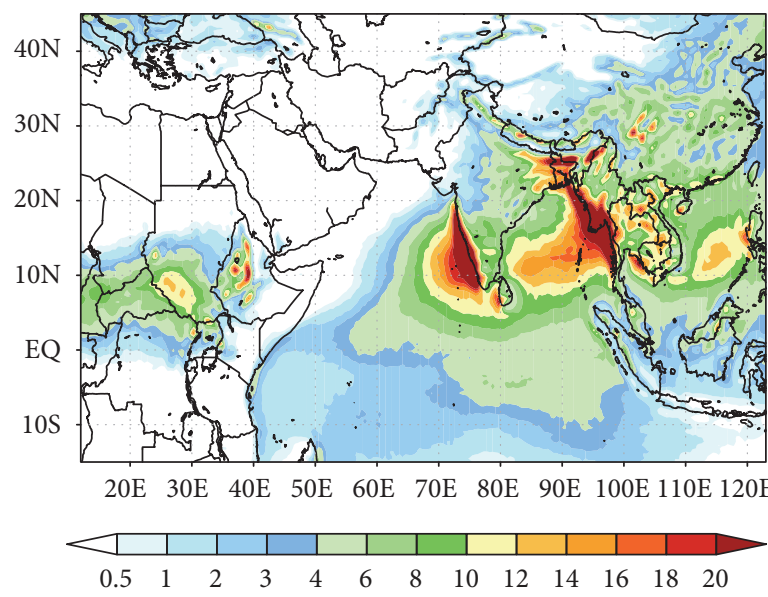

(c) SSBC_New, JJA

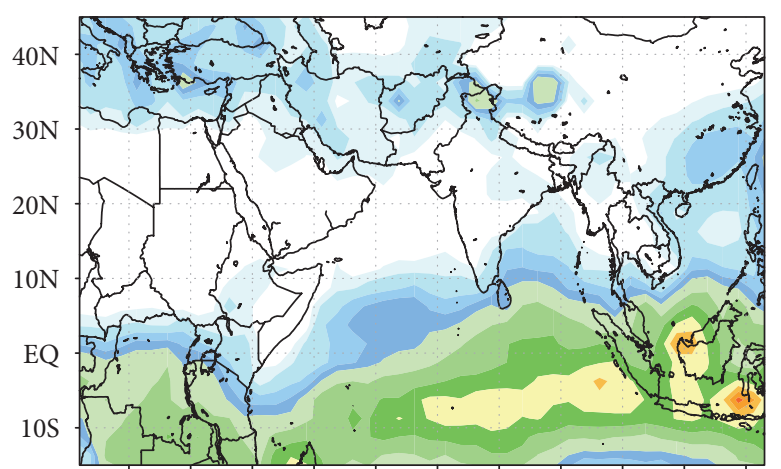

20E $30 \mathrm{E} \quad 40 \mathrm{E} \quad 50 \mathrm{E} \quad 60 \mathrm{E} \quad 70 \mathrm{E} \quad 80 \mathrm{E} \quad 90 \mathrm{E} \quad 100 \mathrm{E} 110 \mathrm{E} 120 \mathrm{E}$

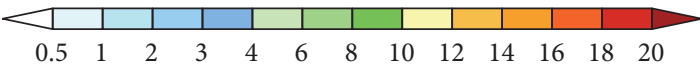

(d) CMAP, DJF

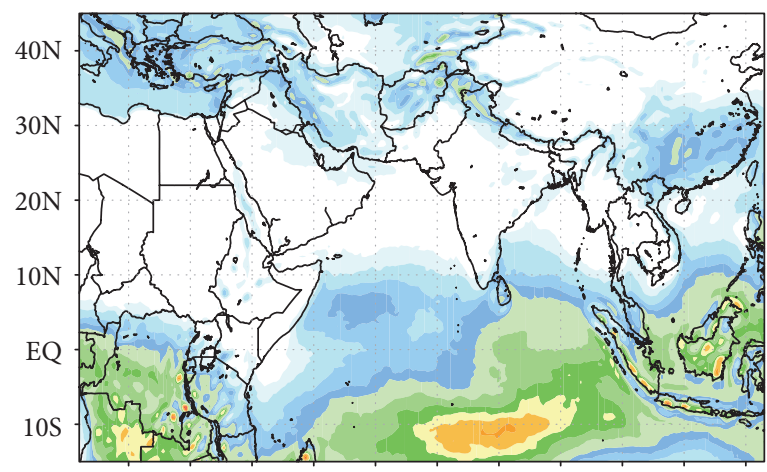

20E $30 \mathrm{E} \quad 40 \mathrm{E} \quad 50 \mathrm{E} \quad 60 \mathrm{E} \quad 70 \mathrm{E} \quad 80 \mathrm{E} \quad 90 \mathrm{E} \quad 100 \mathrm{E} 110 \mathrm{E} 120 \mathrm{E}$

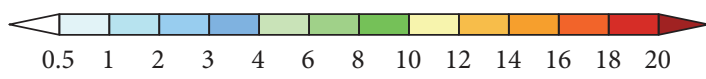

(e) SSBC_Def, DJF

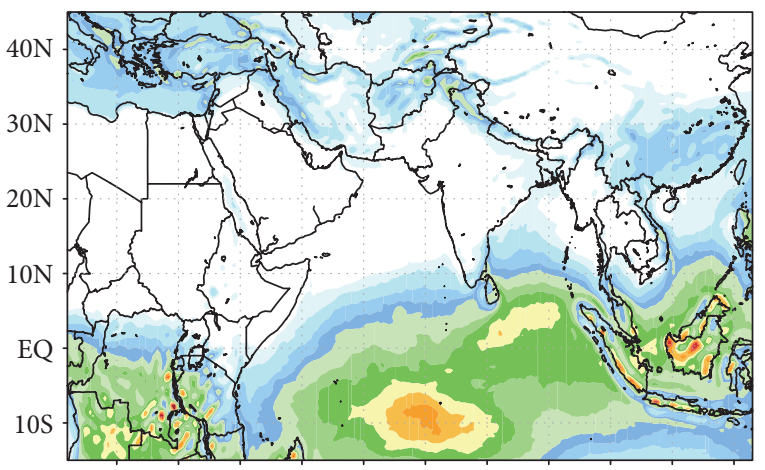

20E $30 \mathrm{E} \quad 40 \mathrm{E} \quad 50 \mathrm{E} \quad 60 \mathrm{E} \quad 70 \mathrm{E} \quad 80 \mathrm{E} \quad 90 \mathrm{E} \quad 100 \mathrm{E} 110 \mathrm{E} 120 \mathrm{E}$

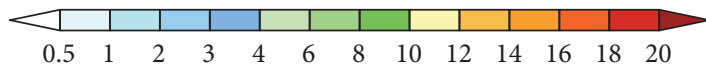

(f) SSBC_New, DJF

FIGURE 4: Seasonally averaged surface precipitation $\left(\mathrm{mm} \mathrm{d}^{-1}\right.$ ) for (a) CMAP, (b) SSBC_Def, and (c) SSBC_New June-July-August (JJA), 1981-1990. (d), (e), and (f) are the same as (a), (b), and (c) but for December-January-February (DJF), 1981-1990, respectively.

dryness depicted in Figures 5(a)-5(d) is due to the boundary conditions used. It is also a positive sign to see that the dryness is minimized in SSBC_New results, which tackles the systematic bias fairly well, as compared with SSBC_Def.
Additionally, the improved performance of SSBC_New may be attributable to the advantage of incorporating a vertical weighting factor [11]. Also, the addition of a divergent wind component to nudging improved the wind and mass balance. 


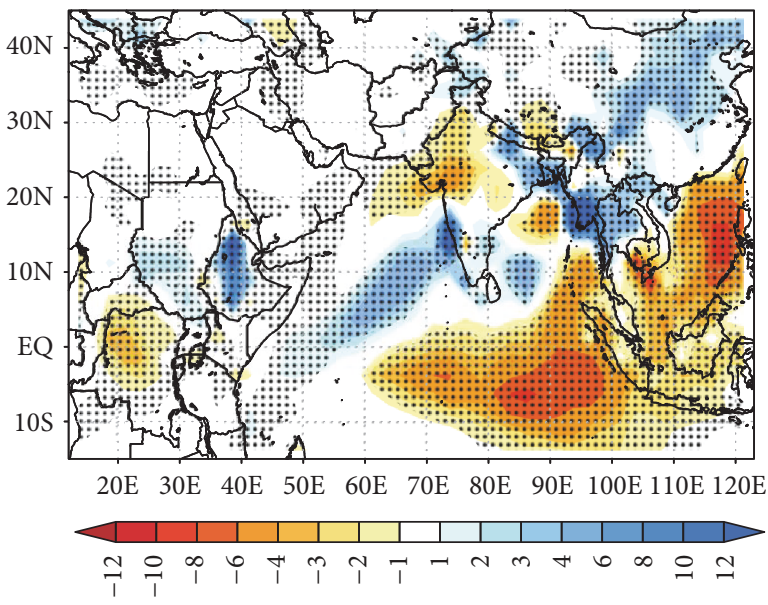

(a) SSBC_Def - CMAP, JJA

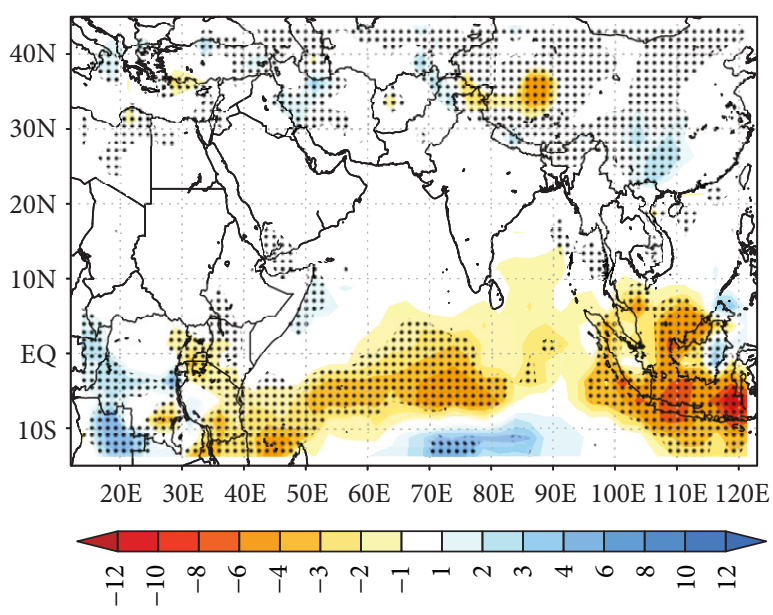

(c) SSBC_Def - CMAP, DJF

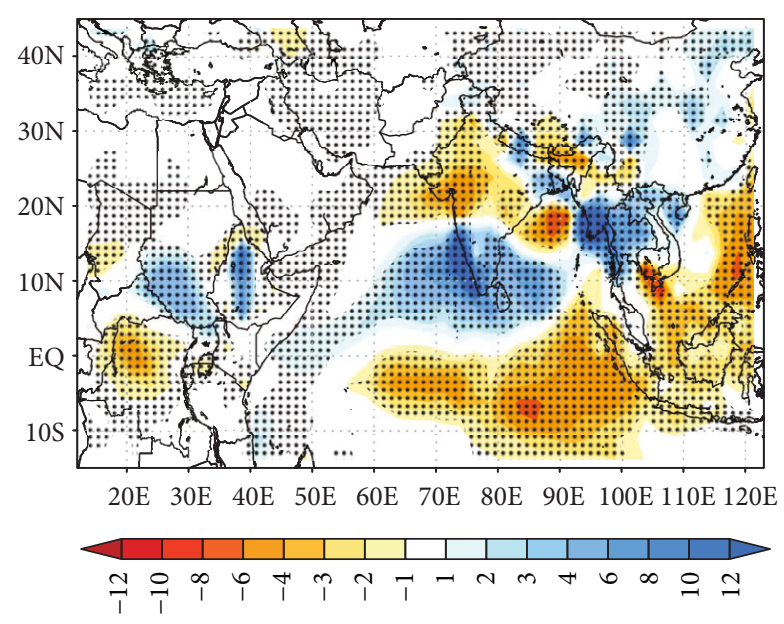

(b) SSBC_New - CMAP, JJA

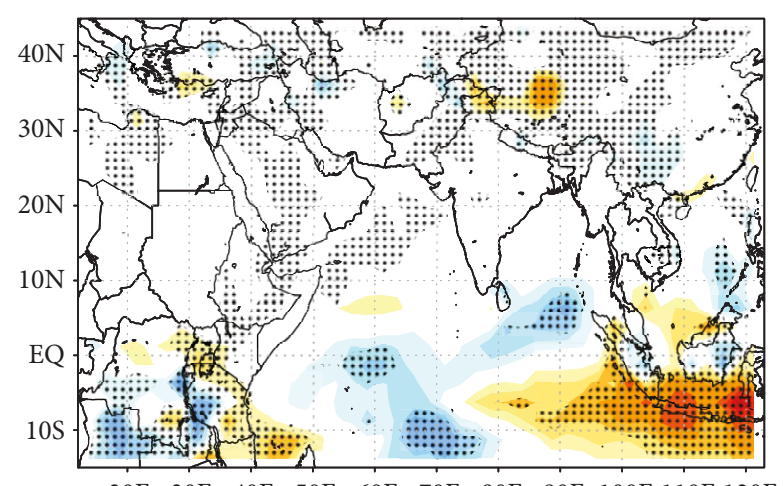

$20 \mathrm{E} \quad 30 \mathrm{E} \quad 40 \mathrm{E} \quad 50 \mathrm{E} \quad 60 \mathrm{E} \quad 70 \mathrm{E} \quad 80 \mathrm{E} \quad 90 \mathrm{E} \quad 100 \mathrm{E} 110 \mathrm{E} 120 \mathrm{E}$

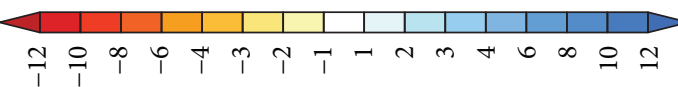

(d) SSBC_New - CMAP, DJF

FIGURE 5: The same as Figure 4 but for the difference of surface precipitation ( $\mathrm{mm} \mathrm{d}^{-1}$ ) of SSBC_Def (b and e) and SSBC_New (c and f) bias relative to CMAP ( $\mathrm{a}$ and $\mathrm{b}$ ) observations. The blue and red colors indicate the wet and dry bias of surface precipitation $\left(\mathrm{mm} \mathrm{d}^{-1}\right)$ over the statistically significant regions (shaded as black dots) at $99 \%$ confidence level.

Thus, due to the natural behavior of ageostrophic winds in the lower atmosphere, it impacted the precipitation simulation of SSBC_New in a positive way, versus SSBC_Def.

In order to enhance our confidence in simulated climatology by both schemes, an additional analysis is performed using APHRO observations (see Figure 6) in JJA and DJF. The findings of this comparison using land only dataset show the same results as those obtained from CMAP comparison which means that SSBC_New is capable of simulating one season and decadal seasonal climatology fairly well.

Figure 7 presents the seasonal variation of surface precipitation climatology for the CORDEX-SA and its subdomain regions. Comparison of CMAP observations with both SSBC types shows that SSBC_Def underestimated the seasonal variation of surface precipitation throughout the series, while SSBC_New exhibited a close approximation to the observed seasonality. Exception is found for WG where SSBC_Def performs well than SSBC_New. It is also important to mention that CMAP observation data are greatly dependent on the quality of the input data. However, it is encouraging to see that SSBC_New shows surface precipitation seasonality closer to CMAP than SSBC_Def.

Figure 8(a) shows the comparison of RMSE for annual precipitation $\left(\mathrm{mm} \mathrm{d}^{-1}\right)$ from 1981 to 1990 for CORDEX-SA. It is evident from the score of errors that the SSBC_Def has a higher error rate than SSBC_New throughout this period. Furthermore, the mean of 10-year RMSE is shown for both SSBC methods (see Figure 8(b)), with standard deviations indicated by the error bars, to differentiate the statistics in both schemes. It is confirmed from the time-series analysis that using the new scheme for the original longterm CORDEX-SA (1980-2005) experiment would be more reasonable.

The SSBC method described by Kanamaru and Kanamitsu [30] considers, by correction, the damping of the longwave spectral coefficients of zonal and meridional wind. SSBC reduces the perturbations of the average regional temperature and humidity to zero. It also corrects the surface 


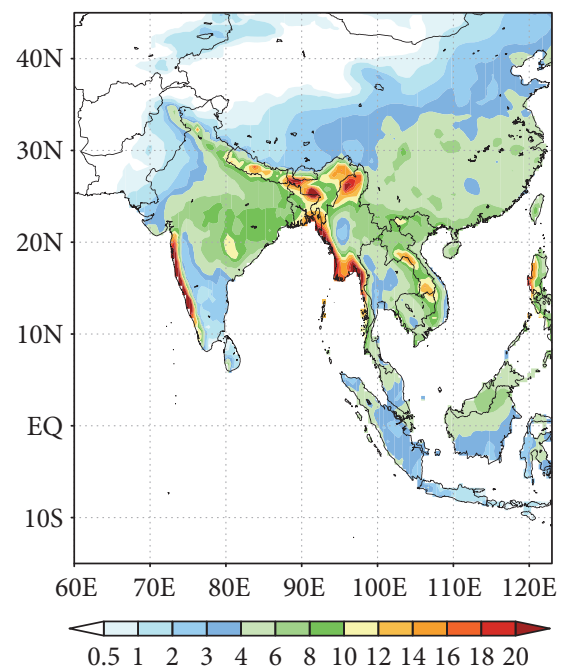

(a) APHRO, JJA

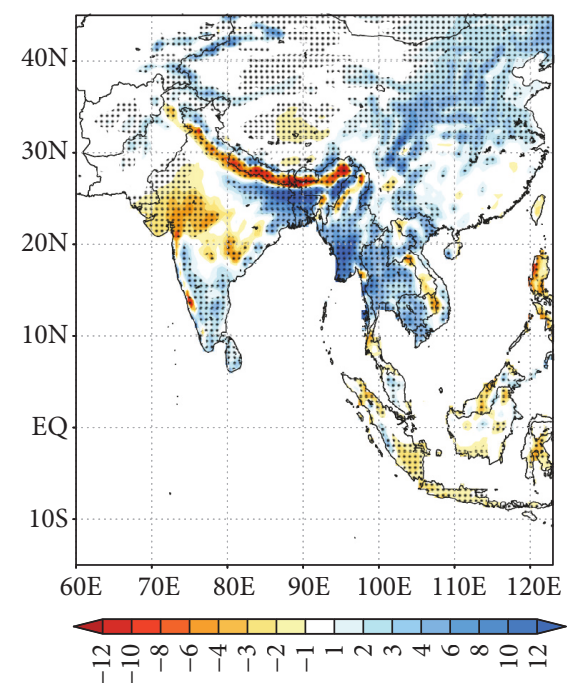

(b) SSBC_Def, APHRO, JJA

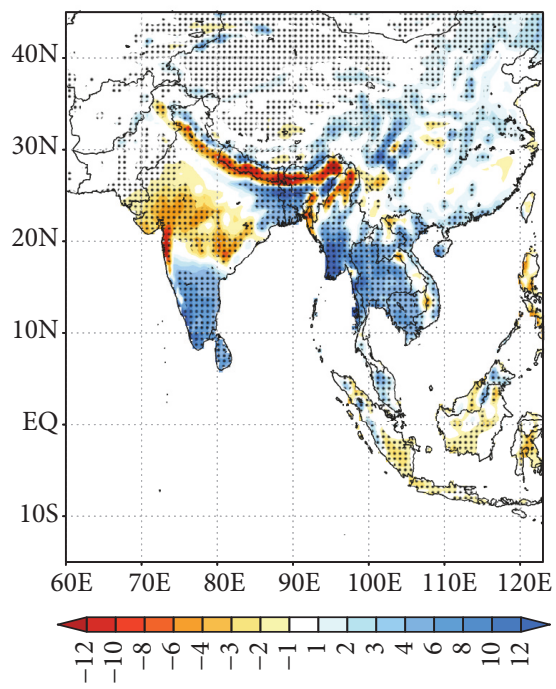

(c) SSBC_New, APHRO, JJA

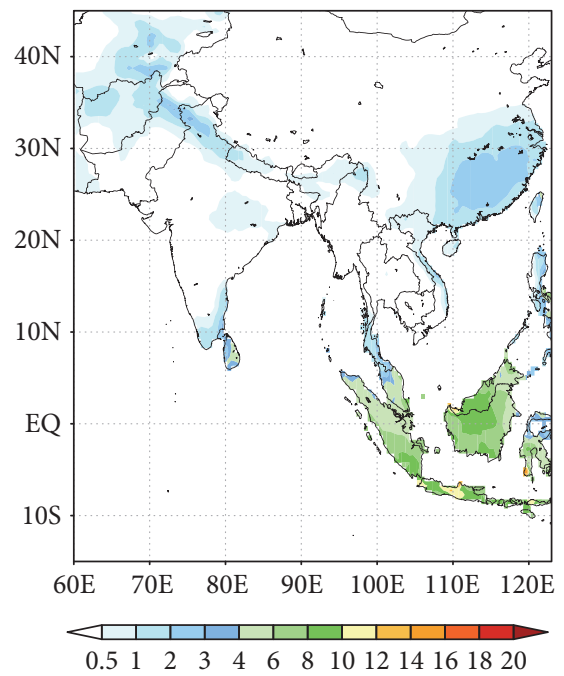

(d) APHRO, DJF

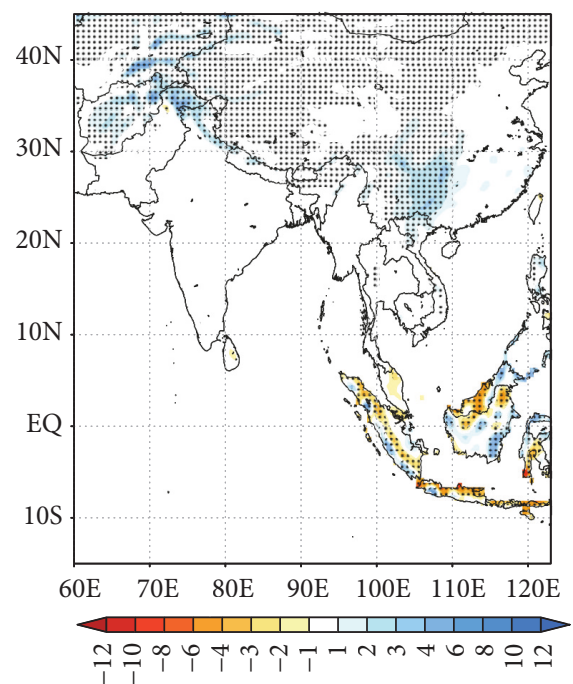

(e) SSBC_Def, APHRO, DJF

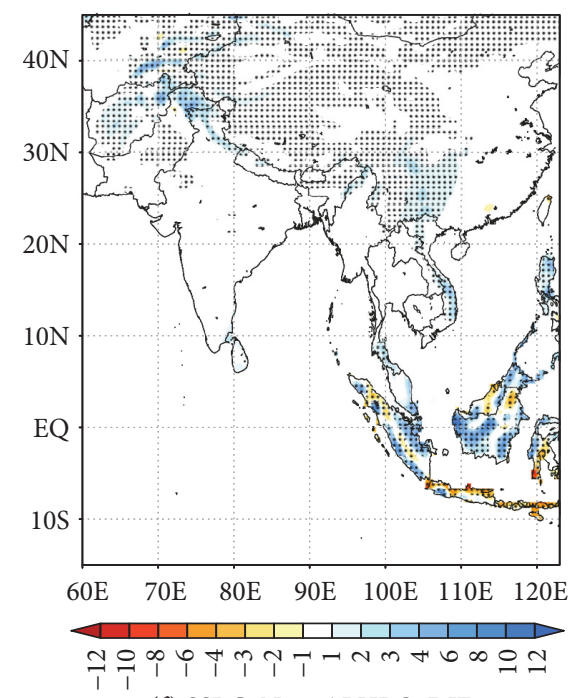

(f) SSBC_New, APHRO, DJF

Figure 6: The same as Figures 4(a), 4(d), 5(b), 5(c), 5(e), and 5(f) but with bias relative to APHRODITE 1981-1990 surface precipitation $\left(\mathrm{mm} \mathrm{d}^{-1}\right)$. 

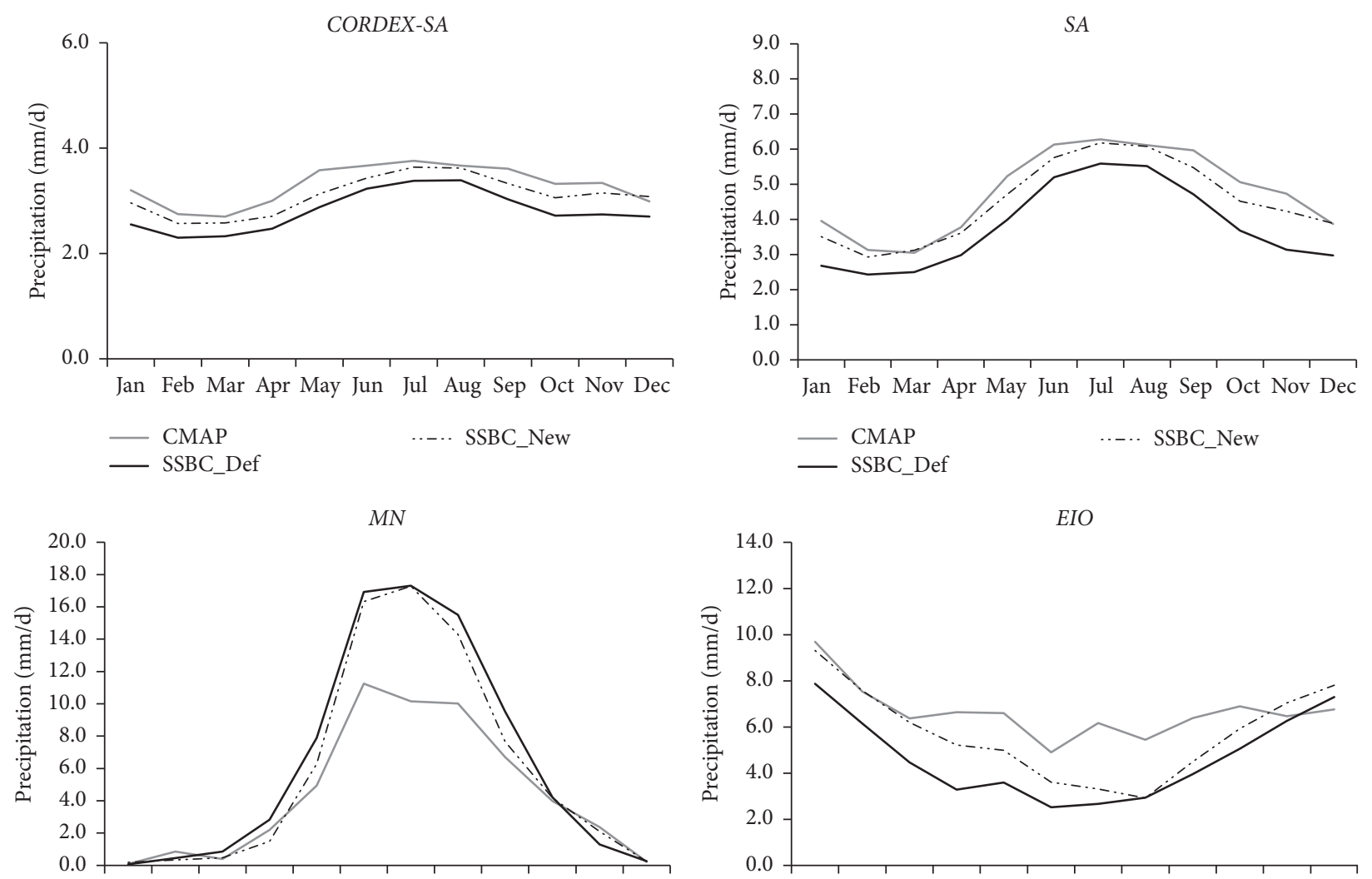

Jan Feb Mar Apr May Jun Jul Aug Sep Oct Nov Dec

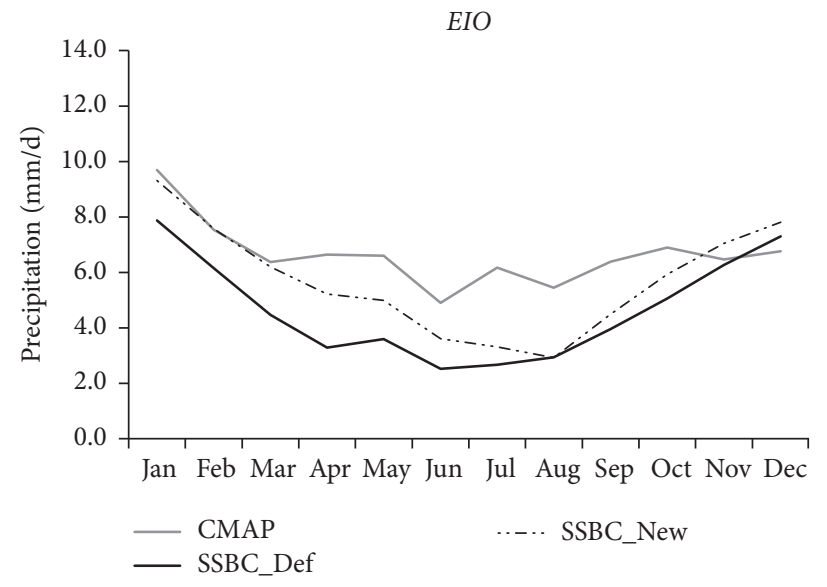

— CMAP
— SSBC_Def

..... SSBC_New

— CMAP
— SSBC_Def

$W G$

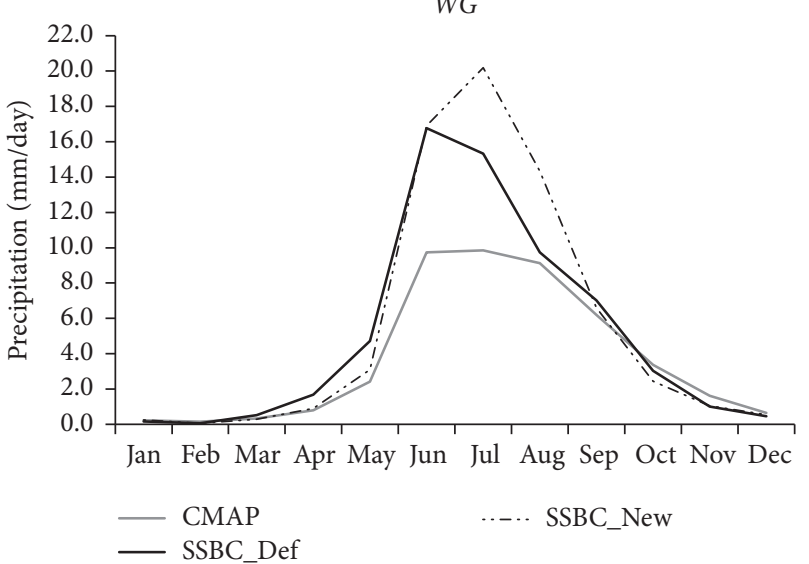

FIGURE 7: Comparison of seasonal variabilities of surface precipitation $\left(\mathrm{mm} \mathrm{d}^{-1}\right)$ among CMAP (grey solid line), SSBC_Def (black solid line), and SSBC_New (black dot dash line) for the CORDEX-SA and its subdomains for years 1981-1990.

pressure between the RCM and the global field. Therefore, further climatology simulated by both SSBC methods is evaluated for the abovementioned variables. Figure 9 and Table 5 present the seasonal climatology of the wind vector and relative humidity at $850 \mathrm{hPa}$ for both SSBC methods against ERA-interim. The results of JJA wind vectors show a similar wind bias for both SSBC simulations (see Figures 9(b) and 9(c)) over the Eastern and WG of India and MN, while a more obvious difference is observed in the relative humidity (RH) given by each method. SSBC_Def greatly underestimated the $\mathrm{RH}$ over the eastern Indian Ocean, whereas there is relatively less dry bias in the SSBC_New simulations. Another underestimation of SSBC_Def over the South China Sea is also minimized by the SSBC_New scheme. These underestimations observed for RH in SSBC_Def can be associated with the dry biases seen in Figure 5(a). This default method shows less precipitation owing to the limited moisture supply in this region (along with systematic bias 


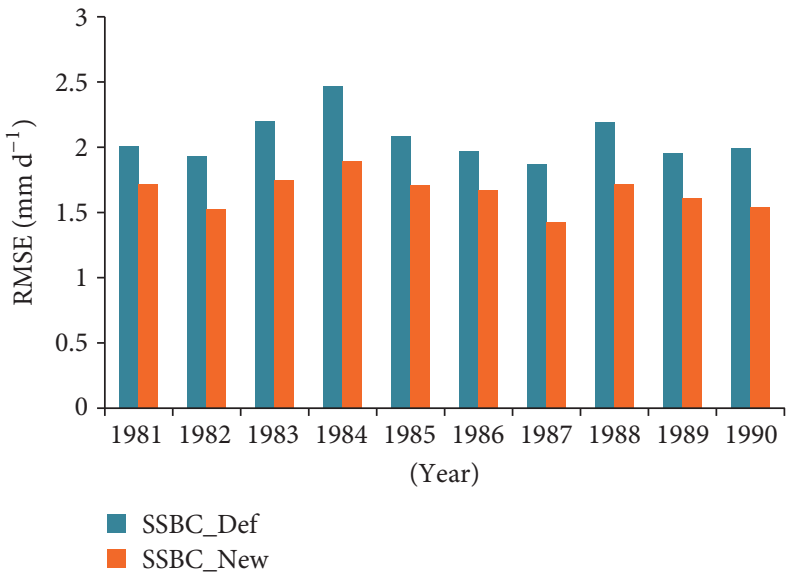

(a)

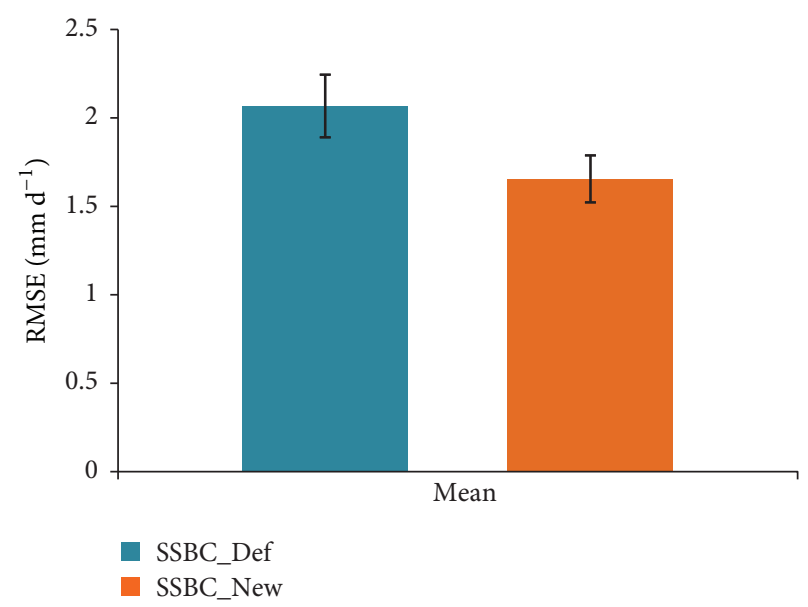

(b)

FIgURE 8: (a) Time series of the root-mean-square error (RMSE) of annual surface precipitation ( $\mathrm{mm} \mathrm{d}^{-1}$ ) from SSBC_Def (blue) and SSBC_New (orange) for CORDEX-SA compared with CMAP observation. (b) Mean of RMSE for 10-year period for both SSBC_Def and SSBC_New. The black error bars represent the standard deviation of both schemes from their mean value.

TABLE 4: Comparisons of the root-mean-square error (RMSE) and spatial correlation (SCORR) of SSBC_Def and SSBC_New summer (JJA) and winter (DJF) surface precipitation $\left(\mathrm{mm} \mathrm{d}^{-1}\right)$ climatology with CMAP observations for 1981-1990 (the bold parts represent the best correlation and least error).

\begin{tabular}{lcccc}
\hline \multirow{2}{*}{ Domain } & \multicolumn{2}{c}{ SSBC_Def } & \multicolumn{2}{c}{ SSBC_New } \\
& RMSE & SCORR & RMSE & SCORR \\
\hline$J J A$ & & & & \\
CORDEX-SA & 3.022 & 0.720 & $\mathbf{2 . 9 2 6}$ & $\mathbf{0 . 7 6 4}$ \\
SA & 4.045 & 0.606 & $\mathbf{4 . 0 2 4}$ & $\mathbf{0 . 6 5 0}$ \\
MN & 10.565 & $\mathbf{0 . 6 7 8}$ & $\mathbf{1 0 . 3 3 2}$ & 0.607 \\
EIO & 3.929 & 0.454 & $\mathbf{3 . 0 0 8}$ & $\mathbf{0 . 8 7 3}$ \\
WG & $\mathbf{8 . 9 7 7}$ & $\mathbf{0 . 9 1 1}$ & 13.138 & 0.907 \\
\hline DJF & & & & \\
CORDEX-SA & 1.883 & 0.832 & $\mathbf{1 . 7 6 0}$ & $\mathbf{0 . 8 6 0}$ \\
SA & 2.193 & $\mathbf{0 . 8 6 3}$ & $\mathbf{2 . 0 1 4}$ & 0.854 \\
MN & $\mathbf{0 . 2 5 3}$ & $\mathbf{0 . 6 2 8}$ & 0.275 & 0.415 \\
EIO & 2.836 & $\mathbf{0 . 5 2 7}$ & $\mathbf{2 . 3 5 8}$ & 0.512 \\
WG & 0.084 & 0.971 & $\mathbf{0 . 0 6 1}$ & $\mathbf{0 . 9 7 3}$ \\
\hline
\end{tabular}

from boundary conditions). Alternatively, SSBC_New shows less dry bias owing to the large $\mathrm{RH}$ present in the region (see Figure 9(c)).

Similarly, the surface precipitation wet biases observed in Figures 5(a) and 5(b) along the Eastern and WG of India and $\mathrm{MN}$ may be associated with an overestimated wind vector near these regions by both SSBC methods (see Figures 9(b) and $9(\mathrm{c})$ ). These overestimated winds, directed from the ocean towards the land, are assumed to hit the mountains of these regions, causing more surface precipitation than the observed. The DJF analysis of wind vectors in SSBC_Def simulations (see Figure 9(e)) shows overestimations around $70^{\circ} \mathrm{E}$ to $90^{\circ} \mathrm{E}$ directed towards the south, which explains the wet bias observed in Figure 5(c) over this region.
Table 5 presents the basic RMSE and SCORR of the wind vectors and $\mathrm{RH}$ for both schemes. The statistical scores of both variables (wind vector and $\mathrm{RH}$ ) are higher for the SSBC_New than SSBC_Def method in JJA and DJF. The mechanism considered to be behind the improved score of the new scheme can be attributed to the introduction of full wind nudging, which allowed the ageostrophic wind to behave as in nature. This leads the model to simulate wind, surface precipitation, and RH more accurately during the process of downscaling. SSBC_Def applies nudging only to the rotational wind component, which is considered insufficient to simulate the abovementioned variables for South Asia.

Figure 10 presents the geopotential height (HGT) and air temperature (TMP) at $850 \mathrm{hPa}$ (Figures $10(\mathrm{a})$ and $10(\mathrm{~d})$ ) for the ERA-interim and the SSBC difference from ERA-interim as JJA (Figures 10(b) and 10(c)) and DJF (Figures 10(e) and 10(f)). A positive HGT bias is seen at lower latitudes for JJA in both SSBC methods. At higher latitudes, SSBC_Def shows a slightly more negative bias in Central Asia than SSBC_New. Specifically, Turkmenistan $\left(59.55^{\circ} \mathrm{E}\right.$ and $\left.38.96^{\circ} \mathrm{N}\right)$ shows a negative HGT bias in both SSBC methods. Consistent with JJA, DJF also shows a positive bias at lower latitudes and a negative bias in Central Asia. In DJF, the negative bias is more obvious in SSBC_New climatology than SSBC_Def. The basic score (Table 5) shows that SSBC_New has smaller RMSE scores than SSBC_Def in JJA, which has higher SCORR. In DJF, SSBC_Def overall shows better results than SSBC_New.

The TMP results for JJA show a warm bias over Turkmenistan for both SSBC methods. Another warm bias is observed over Central Africa, which is overestimated more by SSBC_Def than SSBC_New. A third warm bias is observed in SSBC_Def simulations that covered the midwestern parts of Pakistan and is further extended to the east, towards central India in the SSBC_New simulations. One possible reason for observing the warm bias in both schemes (especially over Turkmenistan) is the known positive temperature bias in 


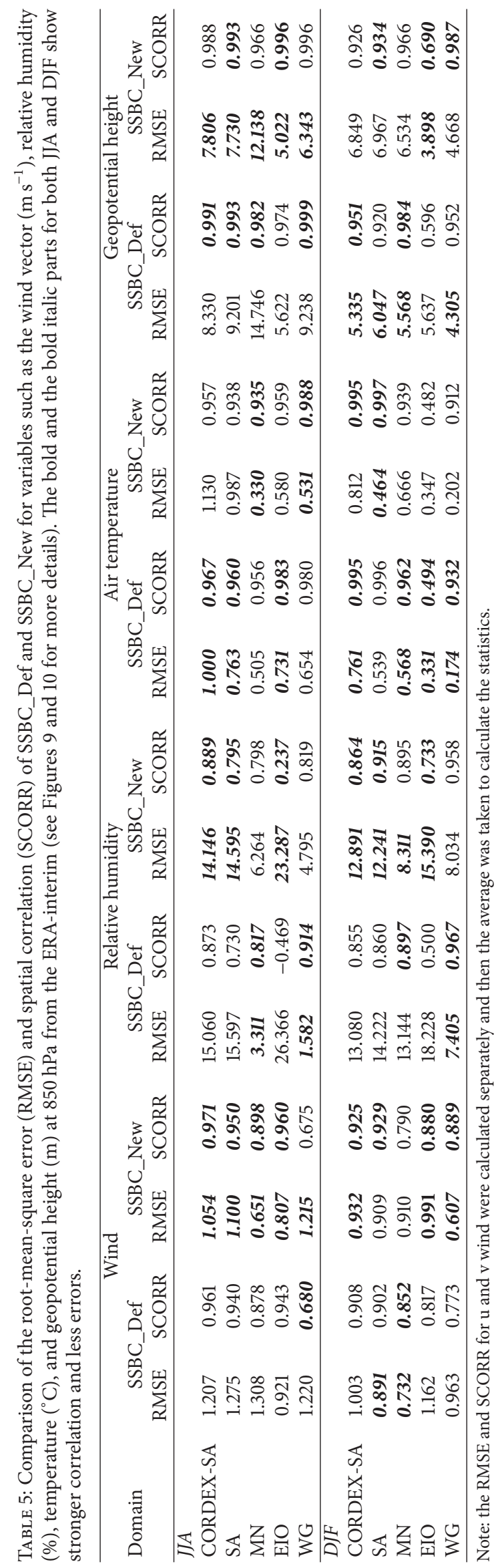




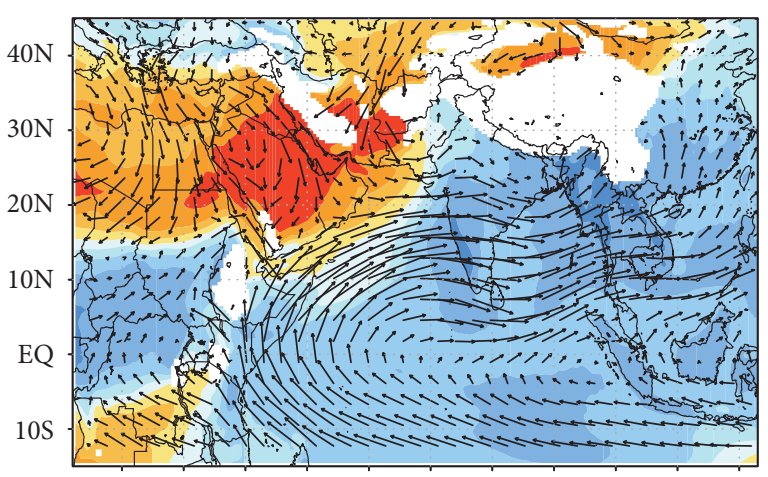

20E $30 \mathrm{E} \quad 40 \mathrm{E} \quad 50 \mathrm{E} \quad 60 \mathrm{E} \quad 70 \mathrm{E} \quad 80 \mathrm{E} \quad 90 \mathrm{E} \quad 100 \mathrm{E} 110 \mathrm{E} 120 \mathrm{E}$

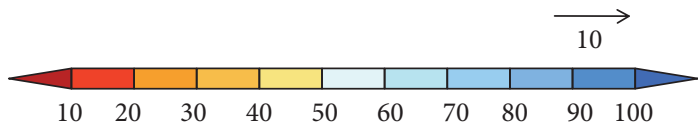

(a) ERA_interim, JJA

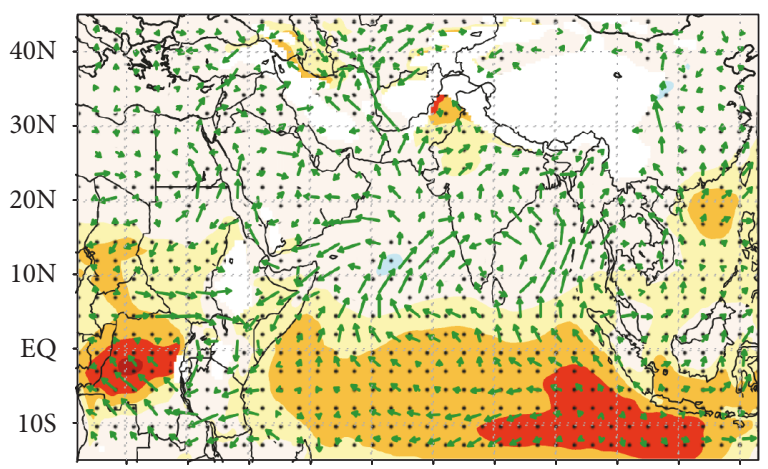

$20 \mathrm{E} \quad 30 \mathrm{E} \quad 40 \mathrm{E} \quad 50 \mathrm{E} \quad 60 \mathrm{E} \quad 70 \mathrm{E} \quad 80 \mathrm{E} \quad 90 \mathrm{E} \quad 100 \mathrm{E} 110 \mathrm{E} 120 \mathrm{E}$

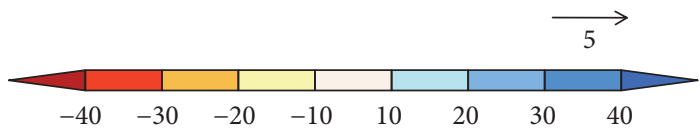

(b) SSBC_Def, ERA, JJA

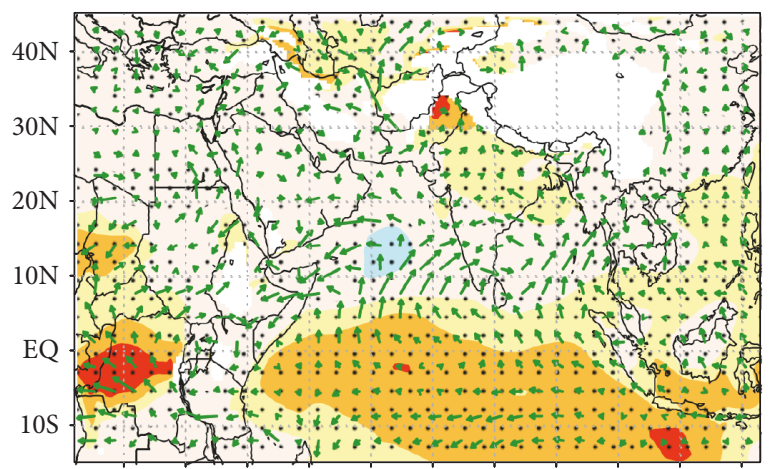

$20 \mathrm{E} \quad 30 \mathrm{E} \quad 40 \mathrm{E} \quad 50 \mathrm{E} \quad 60 \mathrm{E} \quad 70 \mathrm{E} \quad 80 \mathrm{E} \quad 90 \mathrm{E} \quad 100 \mathrm{E} 110 \mathrm{E} 120 \mathrm{E}$

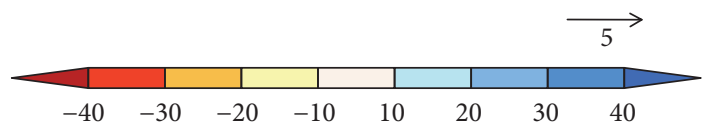

(c) SSBC_New, ERA, JJA

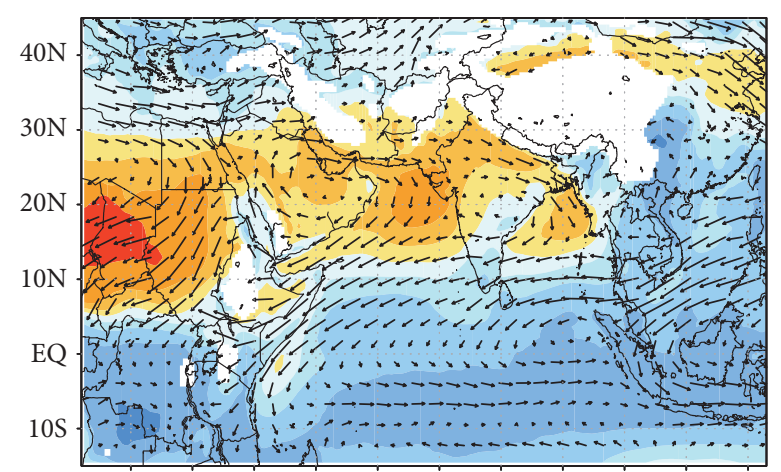

$20 \mathrm{E} \quad 30 \mathrm{E} \quad 40 \mathrm{E} \quad 50 \mathrm{E} \quad 60 \mathrm{E} \quad 70 \mathrm{E} \quad 80 \mathrm{E}$ 90E $100 \mathrm{E} 110 \mathrm{E} 120 \mathrm{E}$

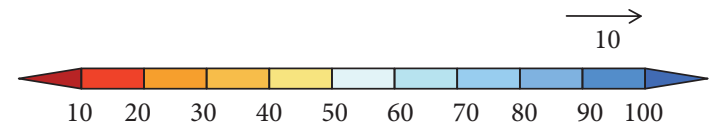

(d) ERA_interim, DJF

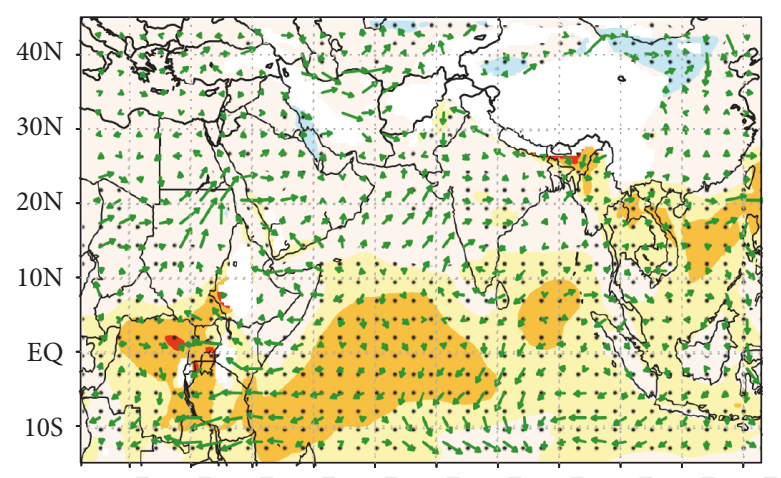

20E $30 \mathrm{E} \quad 40 \mathrm{E} \quad 50 \mathrm{E} \quad 60 \mathrm{E} \quad 70 \mathrm{E} \quad 80 \mathrm{E} \quad 90 \mathrm{E} \quad 100 \mathrm{E} 110 \mathrm{E} 120 \mathrm{E}$

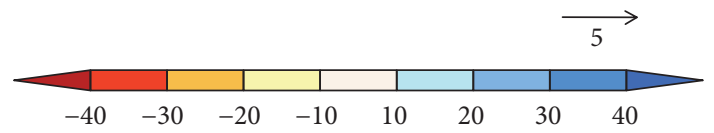

(e) SSBC_Def, ERA, DJF

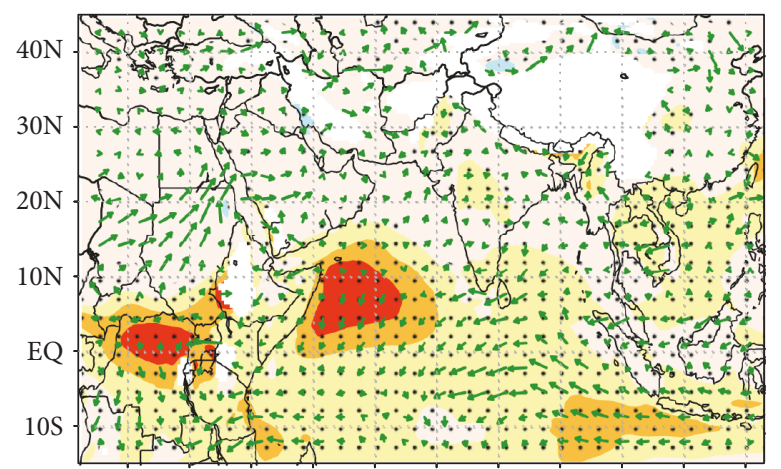

$20 \mathrm{E} \quad 30 \mathrm{E} \quad 40 \mathrm{E} \quad 50 \mathrm{E} \quad 60 \mathrm{E} \quad 70 \mathrm{E} \quad 80 \mathrm{E} \quad 90 \mathrm{E} \quad 100 \mathrm{E} 110 \mathrm{E} 120 \mathrm{E}$

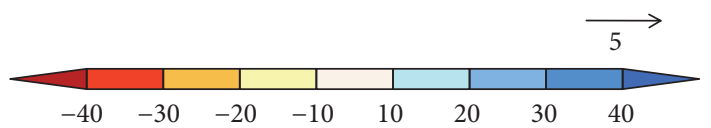

(f) SSBC_New, ERA, DJF

FIGURE 9: Wind vector $\left(850 \mathrm{hPa} ; \mathrm{m} \mathrm{s}^{-1}\right)$ and relative humidity (\%) difference between SSBC_Def (b and e) and SSBC_New (c and f) from ERA_interim reanalysis for JJA and DJF, 1981-1990. The red color is for dry and the blue color is for wet bias of relative humidity. The statistically significant regions at $99 \%$ confidence level shaded as black dots. 


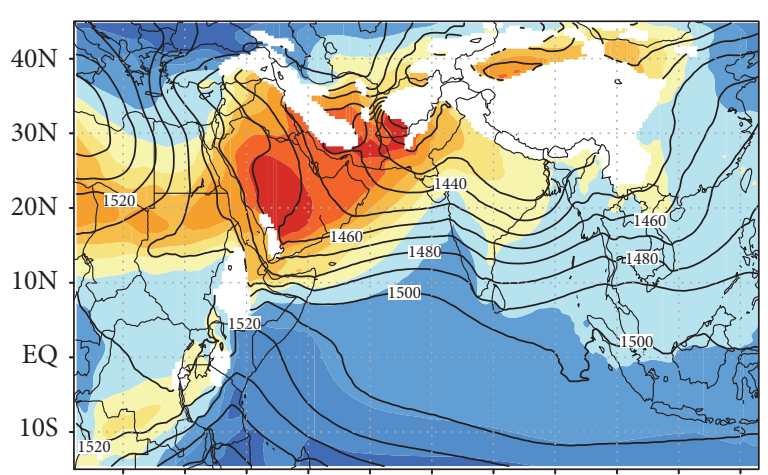

20E $30 \mathrm{E} \quad 40 \mathrm{E} \quad 50 \mathrm{E} \quad 60 \mathrm{E} \quad 70 \mathrm{E} \quad 80 \mathrm{E}$ 90E $100 \mathrm{E} 110 \mathrm{E} 120 \mathrm{E}$

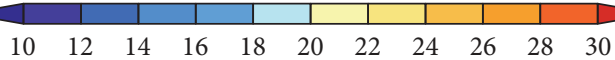

(a) ERA_interim,JJA

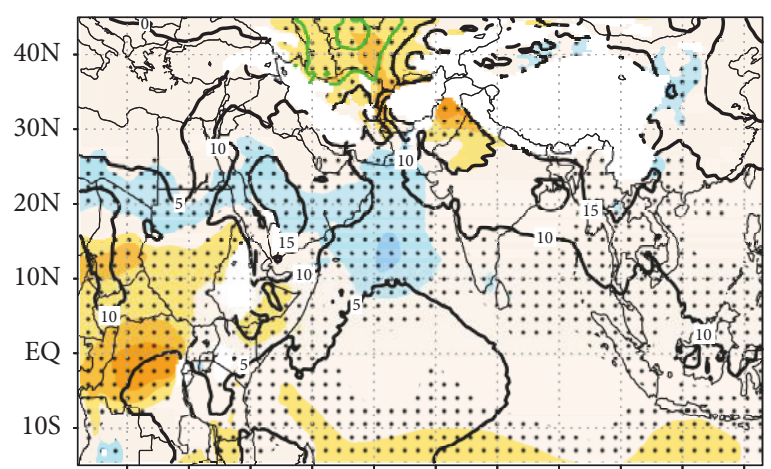

20E $30 \mathrm{E} \quad 40 \mathrm{E} \quad 50 \mathrm{E} \quad 60 \mathrm{E} \quad 70 \mathrm{E} \quad 80 \mathrm{E} \quad 90 \mathrm{E} \quad 100 \mathrm{E} 110 \mathrm{E} 120 \mathrm{E}$

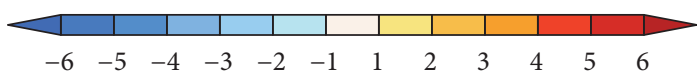

(b) SSBC_Def, ERA, JJA

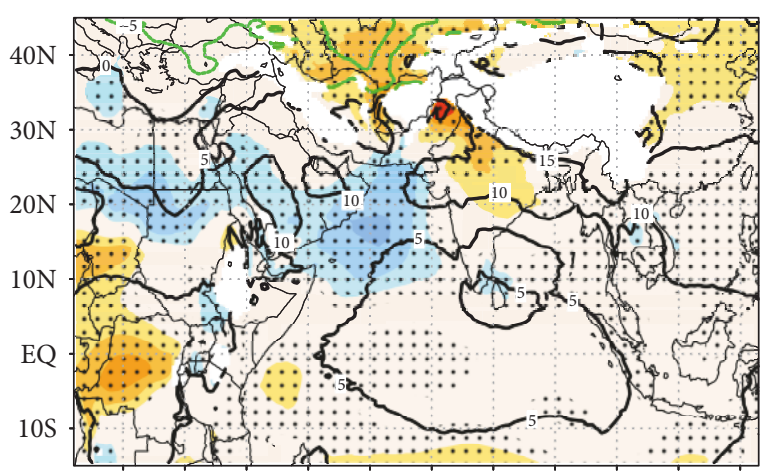

$20 \mathrm{E} \quad 30 \mathrm{E} \quad 40 \mathrm{E} \quad 50 \mathrm{E} \quad 60 \mathrm{E} \quad 70 \mathrm{E} \quad 80 \mathrm{E}$ 90E $100 \mathrm{E} 110 \mathrm{E} 120 \mathrm{E}$

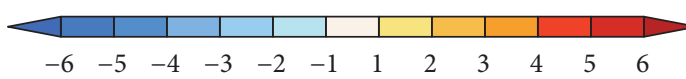

(c) SSBC_New, ERA, JJA

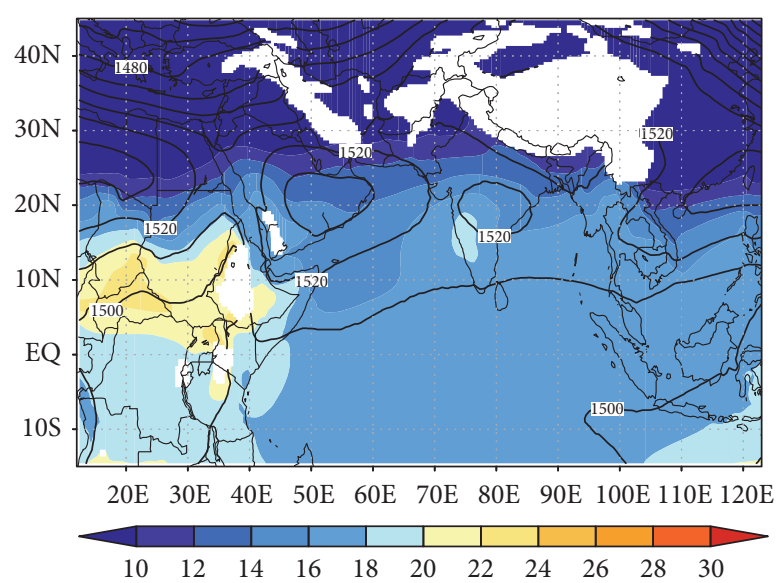

(d) ERA_interim, DJF

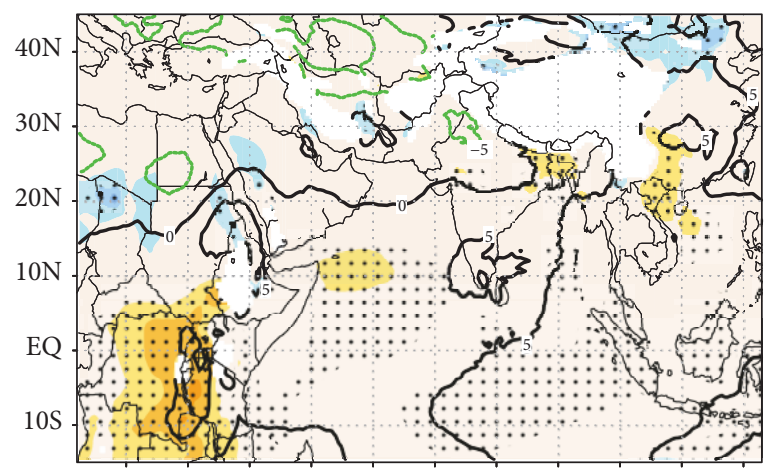

20E $30 \mathrm{E} \quad 40 \mathrm{E} \quad 50 \mathrm{E} \quad 60 \mathrm{E} \quad 70 \mathrm{E} \quad 80 \mathrm{E}$ 90E $100 \mathrm{E} 110 \mathrm{E} 120 \mathrm{E}$

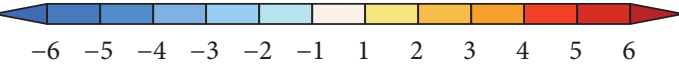

(e) SSBC_Def, ERA, DJF

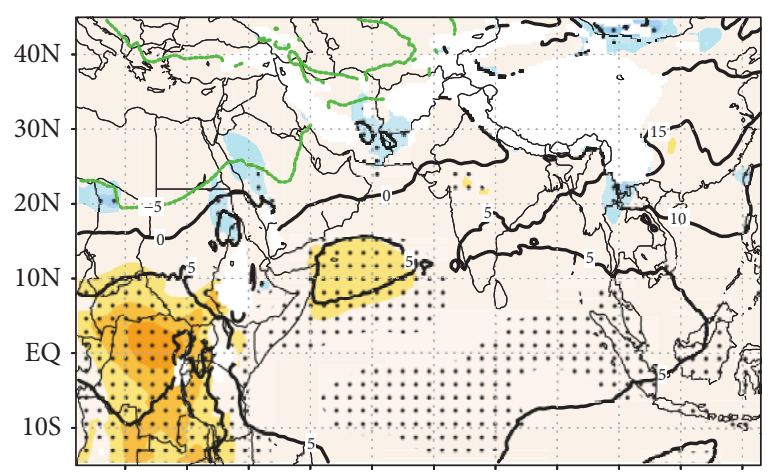

$20 \mathrm{E} \quad 30 \mathrm{E} \quad 40 \mathrm{E} \quad 50 \mathrm{E} \quad 60 \mathrm{E} \quad 70 \mathrm{E} \quad 80 \mathrm{E} \quad 90 \mathrm{E} \quad 100 \mathrm{E} 110 \mathrm{E} 120 \mathrm{E}$

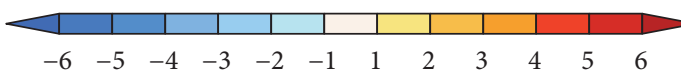

(f) SSBC_New, ERA, DJF

Figure 10: The same as Figure 9 but for the $850 \mathrm{hPa}$ geopotential height (contour, $\mathrm{m}$ ) and the air temperature (shaded, ${ }^{\circ} \mathrm{C}$ ) difference between JJA and DJF, 1981-1990. The red color is for warm and the blue is for cold bias. The statistically significant regions at 99\% confidence level shaded as black dots. The solid black contour is for positive and the green is for negative geopotential height bias, respectively.

ERA-interim, especially under the $850 \mathrm{hPa}$ level towards the Artic regions. Thus, it is possible that both SSBC schemes simulated the TMP fairly well but, due to a systematic bias in ERA-interim, their performances are underestimated. In contrast, a cold bias is observed over the Arabian Sea and extended towards North Africa. SSBC_New overestimated this cold bias to a greater degree than SSBC_Def, along with the overestimation of HGT by SSBC_New over this region. 


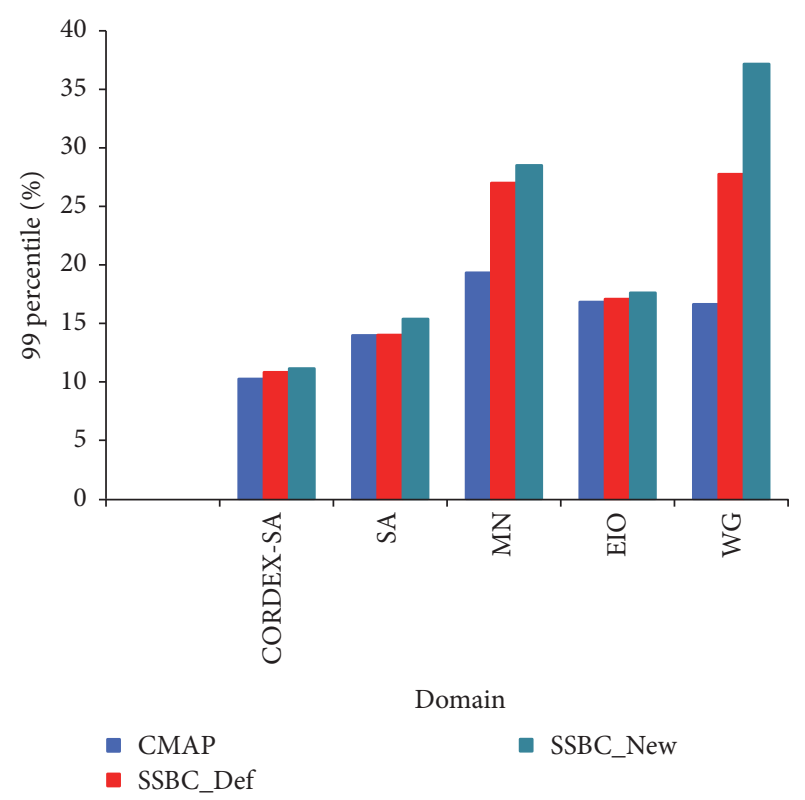

(a)

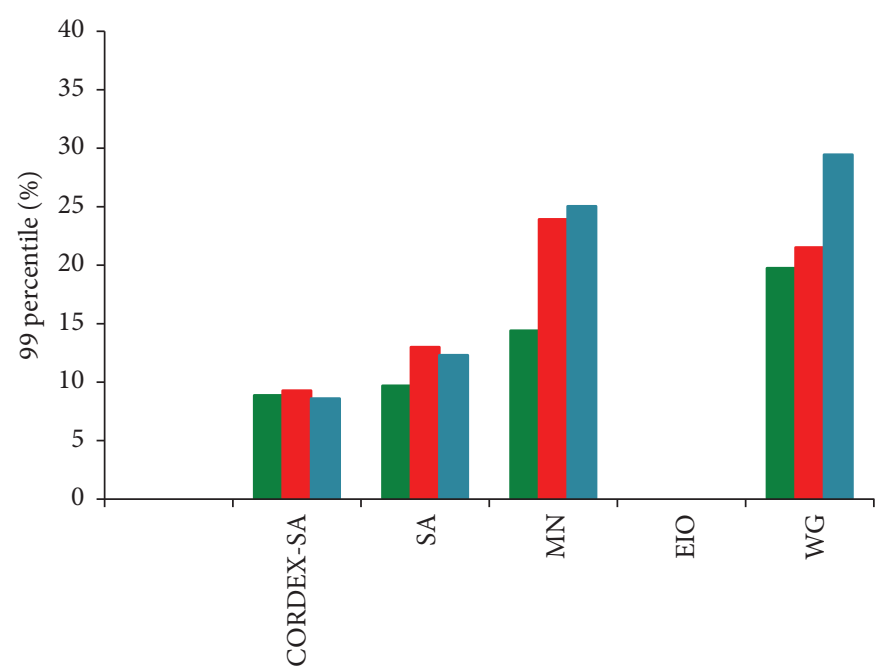

Domain

- SSBC_New

(b)

FIGURE 11: Comparison of 99th percentile surface precipitation over time between SSBC_Def and SSBC_New (1981-1990) for CORDEX-SA and its subdomains with (a) CMAP and (b) APHRODITE surface precipitation (mm day ${ }^{-1}$ ). The $y$-axis units are in \% age.

The TMP results for DJF analysis (Figures $10(\mathrm{e})$ and 10(f)) show a cold bias over northern China, with a positive HGT bias in both methods. The prominent warm bias is observed between approximately $10^{\circ} \mathrm{N}$ and $10^{\circ} \mathrm{S}$ over the African region in both schemes. In conclusion, a negative HGT bias with positive TMP bias is observed in the warm temperate zone $\left(33^{\circ} \mathrm{N}-45^{\circ} \mathrm{N}\right)$ in the summer in both SSBC schemes. A positive HGT bias with positive TMP bias is observed in the tropical zone $\left(10^{\circ} \mathrm{S}-10^{\circ} \mathrm{N}\right)$ in JJA and DJF. The HGT and TMP relationship fluctuated in the subtropical zone $\left(15^{\circ} \mathrm{N}-30^{\circ} \mathrm{N}\right)$. In JJA, it is observed that the positive HGT bias is accompanied by a negative TMP bias. In DJF, it became a negative HGT with a negative TMP bias. Thus, more transition is observed in the subtropical zones, which altered in both seasons. A special case is observed for the JJA over the Pakistan region. It shows a similar pattern to the tropical zone, with positive HGT bias plus positive TMP bias over most of its central parts.

The basic statistical scores for TMP are presented in Table 5, which shows a higher SCORR and lower RMSE for SSBC_Def for both seasons, indicating that the temperature correction applied in both SSBC schemes is more suitable for SSBC_Def which helps in simulating the air temperature at lower atmospheric levels. SSBC_New shows SCORR similar to SSBC_Def in DJF but with higher RMSE.

An additional analysis is performed for the wind vector, RH, HGT, and TMP using the NCEP-CFSR dataset for JJA and DJF of 1981-1990 to enhance our confidence in the findings above. The results of the basic scores are given in Table 6 . The finding of this analysis shows the similar results as those obtained by the comparison with ERA-interim. Thus, it is confirmed from this sensitivity study that the largescale bias reduction features are well simulated in SSBC_Def, which may be one reason why we obtained better scores for TMP and HGT, but this needs further analysis.

Based upon the validation of both SSBC schemes at $850 \mathrm{hPa}$ with ERA-interim and NCEP-CFSR, it is summarized that SSBC_New best captures $\mathrm{RH}$ in both seasons by all subdomains except for WG. Wind is simulated well by SSBC_New in JJA and by SSBC_Def in DJF. For TMP, overall in both seasons, the SCORR is higher in SSBC_Def but RMSE is lower in SSBC_New. Similar results are obtained for HGT for both schemes but only for JJA. For DJF, the performance fluctuates showing no visible trend over the subdomains except for EIO, which is well simulated by SSBC_New.

3.3. Very Heavy Precipitation Analysis (1981-1990). The results for the 10-year very heavy precipitation analysis (99th percentile) for the selected domains are shown in Figures 11(a) and 11(b) and in Table 7, respectively.

Figure 11(a) shows CMAP bars as blue, SSBC_Def as red, and SSBC_New as aqua color. The comparison of SSBC schemes relative to CMAP observations shows closer proximity of 99th percentiles when the domain size is large (see, e.g., CORDEX-SA, SA, and EIO). For smaller domains (such as $\mathrm{MN}$ and $\mathrm{WG}$ ), the 99th percentiles are overestimated by both schemes. The overestimations are larger in SSBC_New than SSBC_Def. One possible reason of the overestimation by both schemes can be the coarse resolution of CMAP over the smaller domains, which cannot capture the very heavy precipitation events.

Figure 11(b) shows the comparison of SSBC schemes relative to APHRO. The bar color for APHRO is green while the colors for both schemes are the same as those in Figure 11(a). Please note that all the ocean parts for this figure are masked to eliminate any inconsistency between model and 


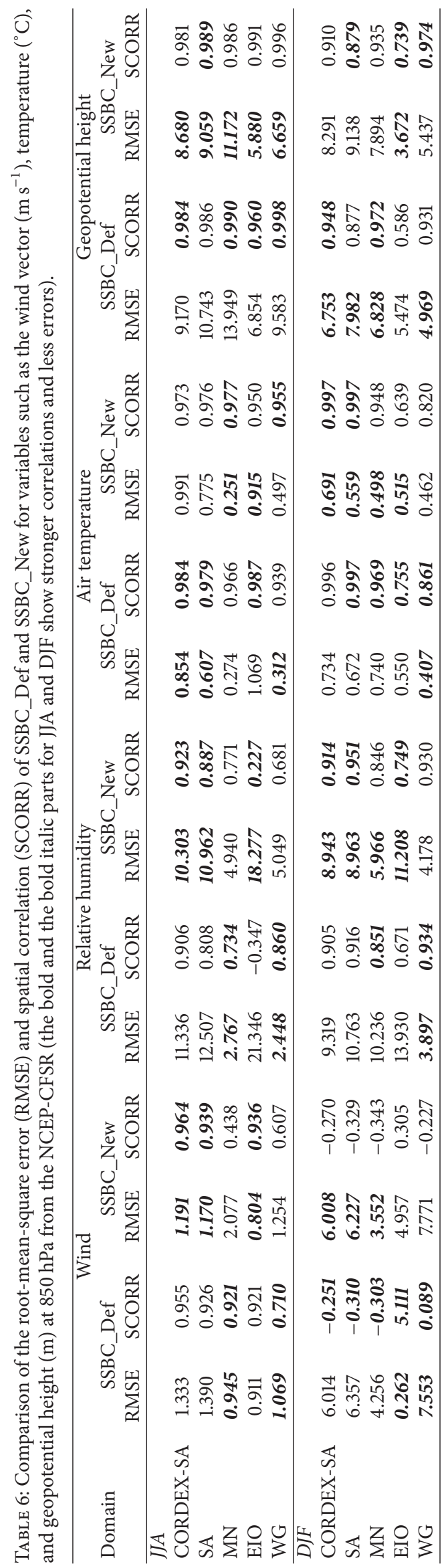


TABLE 7: 99th percentile statistics of surface precipitation for SSBC_Def and SSBC_New against CMAP and APHRODITE, 1981-1990. Units are in \% age.

(a) Land + ocean (1981-1990)

\begin{tabular}{lccc}
\hline Domain & CMAP & SSBC_Def & SSBC_New \\
\hline CORDEX-SA & 10.245 & 10.814 & 11.136 \\
SA & 13.966 & 14.001 & 15.376 \\
MN & 19.323 & 26.996 & 28.499 \\
EIO & 16.823 & 17.069 & 17.612 \\
WG & 16.629 & 27.744 & 37.166 \\
\hline
\end{tabular}

(b) Land only (1981-1990)

\begin{tabular}{lccc}
\hline Domain & APHRO & SSBC_Def & SSBC_New \\
\hline CORDEX-SA & 8.800 & 9.205 & 8.535 \\
SA & 9.620 & 12.926 & 12.235 \\
MN & 14.330 & 23.858 & 24.968 \\
EIO & NA & NA & NA \\
WG & 19.678 & 21.444 & 29.380 \\
\hline
\end{tabular}

NA: not applicable.

observation coverage (e.g., the absence of EIO in comparison). The results of this analysis show almost similar findings as those obtained for Figure 11(a) for larger domains. However, for smaller domains, the results look more promising than CMAP for WG. The comparison between SSBC schemes shows that, for CORDEX-SA domain, the SSBC_New shows more similar percentiles as APHRO but when moving to subdomain levels, SSBC_Def takes over the place of SSBC_New in performance. There are a number of probable reasons for such behavior. The one prominent reason of SSBC_New's overestimated percentile can be related to the wet bias observed in JJA seasonal climatology (see Figures 4, 5, and 6) over WG. It is also crucial to mention the known bias in the observation data as well. APHRO is known for the heterogeneity of its spatial and temporal coverage (especially over India).

Thus, while keeping the limitations of both datasets in view, the results of this analysis can lead us to multiple interpretations. These include (i) the effect of observation resolution, (ii) the size of domain, and (iii) the land-ocean coverage. Overall, the summation of this analysis can be in the favor the SSBC_Def percentiles when coarse observation data is used and vice versa for SSBC_New.

\section{Summary and Concluding Remarks}

The objective of this sensitivity study was to fulfill the purpose of the CORDEX experiment, which emphasized the use of improved downscaling techniques and their applications in impact studies $[31,32]$. This sensitivity study applies the scaleselective bias correction (SSBC) method of Kanamitsu et al. [10] and Hong and Chang [11], developed for RSM on our domain of CORDEX-SA. The former was named SSBC_Def and the latter SSBC_New. The SSBC_Def method applies (i) nudging only to the rotational wind components and (ii) the vertical weighted factor which was constant (a small coefficient was selected by default). SSBC_New on the other hand (i) applies nudging to the full wind components and (ii) considers the vertically weighted damping coefficients, which vary with the atmospheric levels.

A broad community will use the selected technique; therefore, our purpose was to analyze the strengths and weaknesses of the selected method. This could be achieved by evaluating and quantifying their performance, in analyzing the selected issues or events [33].

Both schemes were assessed in two time frames. One was the short-term summer of 2005, focusing on precipitation, and the second was the climatological experiment, focusing on 10 years (1981-1990) of surface precipitation. The results of these experiments showed that SSBC_New outperformed SSBC_Def in terms of capturing the precipitation on seasonal and climatological scales. The decadal climatology will remain the focus of further analyses. The results of seasonal variability of monthly precipitation and time series of root-mean-square error for annual precipitation further confirmed the advantages of the new scheme.

The new scheme is recommended as a reasonable option when applying dynamic downscaling over South Asia using the regional spectral model (RSM). The improved performance of SSBC_New is attributable to both vorticity nudging, which decreases gradually from higher levels towards the ground surface, and the vertical weighted damping coefficient. Their combined effect helped the RSM model to simulate the surface precipitation more realistically than SSBC_Def. The full wind nudging applied in SSBC_New yields improved results for wind vector and relative humidity at lower tropospheric levels.

SSBC_Def did not show a higher score for surface precipitation, wind, or relative humidity; however, the results of air temperature and geopotential height are notably better than SSBC_New. Thus, SSBC_Def is suitable for air temperature and geopotential height, for the specified time period used in this study, and for the analyzed seasons.

Based on this sensitivity study, it can be concluded that SSBC_New is applicable to CORDEX-SA. It is suggested that this new SSBC method may be independent of domain restrictions, but further analysis is required. The use of the SSBC_New scheme will depend on the user's requirements. In this current research, the improved precipitation, wind pattern, and relative humidity gave satisfactory results, which fulfilled the requirements of preliminary sensitivity experiments. These results suggest that SSBC_New is suitable for reliable long-term analysis and will facilitate future studies.

\section{Conflicts of Interest}

It is declared that there are no conflicts of interest or financial gains regarding the publication of this paper.

\section{Acknowledgments}

Mehwish Ramzan is grateful to the Ministry of Education, Culture, Sports, Science and Technology, Japan (MEXT) for the provision of funding for their higher education in Japan. Thanks are extended to The University of Tokyo for providing 
the best possible computational resources. Special regards are given to the SOUSEI project (Program for Risk Information on Climate Change) for covering travel and other expenses during conferences and workshops attended throughout the research. It is the authors' great privilege to acknowledge the support of Environment Research and Technology Development Fund (S-12) from Japanese Ministry of Environment.

\section{References}

[1] World Bank, Turn Down the Heat: Climate Extremes, Regional Impacts, and the Case for Resilience. A Report for the World Bank by the Potsdam Institute for Climate Impact Research and Climate Analytics, World Bank, Washington, DC, USA, 2013.

[2] H.-M. H. Juang and M. Kanamitsu, "The NMC nested regional spectral model," Monthly Weather Review, vol. 122, no. 1, pp. 326, 1994.

[3] T. P Sabin, R. Krishnan, J. Ghattas et al., "High resolution simulation of the South Asian monsoon using a variable resolution global climate model," Climate Dynamics, vol. 41, no. 1, pp. 173-194, 2013.

[4] S. Gadgil and S. Sajani, "Monsoon precipitation in the AMIP runs," Climate Dynamics, vol. 14, no. 9, pp. 659-689, 1998.

[5] Z. Xu and Z.-L. Yang, "An improved dynamical downscaling method with GCM bias corrections and its validation with 30 years of climate simulations," Journal of Climate, vol. 25, no. 18, pp. 6271-6286, 2012.

[6] H. Von Storch, H. Langenberg, and F. Feser, "A spectral nudging technique for dynamical downscaling purposes," Monthly Weather Review, vol. 128, no. 10, pp. 3664-3673, 2000.

[7] S. Song, J. Tang, and X. Chen, "Impacts of spectral nudging on the sensitivity of a regional climate model to convective parameterizations in East Asia," Acta Meteorologica Sinica, vol. 25, no. 1, pp. 63-77, 2011.

[8] P. Uhe and M. Thatcher, "A spectral nudging method for the ACCESS1.3 atmospheric model," Geoscientific Model Development, vol. 8, no. 6, pp. 1645-1658, 2015.

[9] H. Kanamaru and M. Kanamitsu, "Scale-selective bias correction in a downscaling of global analysis using a regional model," Monthly Weather Review, vol. 135, no. 2, pp. 334-350, 2007.

[10] M. Kanamitsu, K. Yoshimura, Y.-B. Yhang, and S.-Y. Hong, "Errors of interannual variability and trend in dynamical downscaling of reanalysis," Journal of Geophysical Research Atmospheres, vol. 115, no. 17, Article ID D17115, 2010.

[11] S.-Y. Hong and E.-C. Chang, "Spectral nudging sensitivity experiments in a regional climate model," Asia-Pacific Journal of Atmospheric Sciences, vol. 48, no. 4, pp. 345-355, 2012.

[12] A. D. Choudhury and R. Krishnan, "Dynamical response of the South Asian monsoon trough to latent heating from stratiform and convective precipitation," Journal of the Atmospheric Sciences, vol. 68, no. 6, pp. 1347-1363, 2011.

[13] L. Guo, A. G. Turner, and E. J. Highwood, "Impacts of 20th century aerosol emissions on the South Asian monsoon in the CMIP5 models," Atmospheric Chemistry and Physics, vol. 15, no. 11, pp. 6367-6378, 2015.

[14] Y. Gao, H. Wang, and D. Jiang, "An intercomparison of CMIP5 and CMIP3 models for interannual variability of summer precipitation in Pan-Asian monsoon region," International Journal of Climatology, vol. 35, no. 13, pp. 3770-3780, 2015.

[15] H. Annamalai and K. R. Sperber, "South Asian summer monsoon variability in a changing climate," in The Monsoons and
Climate Change, L. M. V. Carvalho and C. Jones, Eds., pp. 2546, Springer, Basel, Switzerland, 2016.

[16] S.-Y. Hong and H.-L. Pan, "Convective trigger function for a mass-flux cumulus parameterization scheme," Monthly Weather Review, vol. 126, no. 10, pp. 2599-2620, 1998.

[17] M. B. Ek, K. E. Mitchell, Y. Lin et al., "Implementation of Noah land surface model advances in the National Centers for Environmental Prediction operational mesoscale Eta model," Journal of Geophysical Research D: Atmospheres, vol. 108, no. 22, article 296, 2003.

[18] M.-D. Chou and M. J. Suarez, "An efficient thermal infrared radiation parameterization for use in general circulation models," NASA Tech. Rep. TM-1994-104606, NASA, Houston, Tex, USA, 1994, Series on Global Modeling and Data Assimilation.

[19] S.-Y. Hong and H.-L. Pan, "Nonlocal boundary layer vertical diffusion in a medium-range forecast model," Monthly Weather Review, vol. 124, no. 10, pp. 2322-2339, 1996.

[20] J. C. Alpert, M. Kanamitsu, P. M. Caplan, J. G. Sela, G. H. White, and E. Kalnay, "Mountain induced gravity wave drag parameterization in the NMC medium-range forecast model," in Proceedings of the 8th Conference on Numerical Weather Prediction, pp. 726-733, American Meteorological Society, Baltimore, Md, USA, 1988.

[21] J. C. Alpert, S.-Y. Hong, and Y.-J. Kim, "Sensitivity of cyclogenesis to lower tropospheric enhancement of gravity wave drag using the environmental modeling center medium range model," in Proceedings of the 11th Conference on Numerical Weather Prediction, pp. 322-323, American Meteorological Society, 1996.

[22] J. Kim and L. Mahrt, "Momentum transport by gravity waves," Journal of the Atmospheric Sciences, vol. 49, no. 9, pp. 735-748, 1992.

[23] M. Kanamitsu, W. Ebisuzaki, J. Woollen et al., "NCEP-DOE AMIP-II reanalysis (R-2)," Bulletin of the American Meteorological Society, vol. 83, no. 11, pp. 1631-1643, 2002.

[24] S. Saha, "NCEP Climate Forecast System Reanalysis (CFSR) Monthly Products, January 1979 to December 2010," Research Data Archive at the National Center for Atmospheric Research, Computational and Information Systems Laboratory, 2010.

[25] A. Yatagai, K. Kamiguchi, O. Arakawa, A. Hamada, N. Yasutomi, and A. Kitoh, "APHRODITE: constructing a long-term daily gridded precipitation dataset for Asia based on a dense network of rain gauges," Bulletin of the American Meteorological Society, vol. 93, no. 9, pp. 1401-1415, 2012.

[26] Y. L. Lin, Mesoscale Dynamics, Cambridge University Press, New York, NY, USA, 2007.

[27] K. Rajendran and A. Kitoh, "Indian summer monsoon in future climate projection by a super high-resolution global model," Current Science, vol. 95, no. 11, pp. 1560-1569, 2008.

[28] H. Wulf, B. Bookhagen, and D. Scherler, "Seasonal precipitation gradients and their impact on fluvial sediment flux in the Northwest Himalaya," Geomorphology, vol. 118, no. 1-2, pp. 1321, 2010.

[29] K. R. Sperber, H. Annamalai, I.-S. Kang et al., "The Asian summer monsoon: an intercomparison of CMIP5 vs. CMIP3 simulations of the late 20th century," Climate Dynamics, vol. 41, no. 9-10, pp. 2711-2744, 2013.

[30] H. Kanamaru and M. Kanamitsu, "Scale-selective bias correction in a downscaling of global analysis using a regional model," PIER Energy Related Environmental Research CEC-500-2005130, California Energy Commission, 2005. 
[31] F. Giorgi, C. Jones, and G. Asrar, "Addressing climate information needs at the regional level: the CORDEX framework," WMO Bulletin, vol. 58, pp. 175-183, 2009.

[32] C. Jones, F. Giorgi, and G. Asrar, "The coordinated regional downscaling experiment: CORDEX, an international downscaling link to CMIP5," CLIVAR Exchanges, vol. 16, no. 2, pp. 34-40, 2011.

[33] W. J. Gutowski Jr., F. Giorgi, B. Timbal et al., "WCRP COordinated Regional Downscaling EXperiment (CORDEX): a diagnostic MIP for CMIP6," Geoscientific Model Development, vol. 9, no. 11, pp. 4087-4095, 2016. 

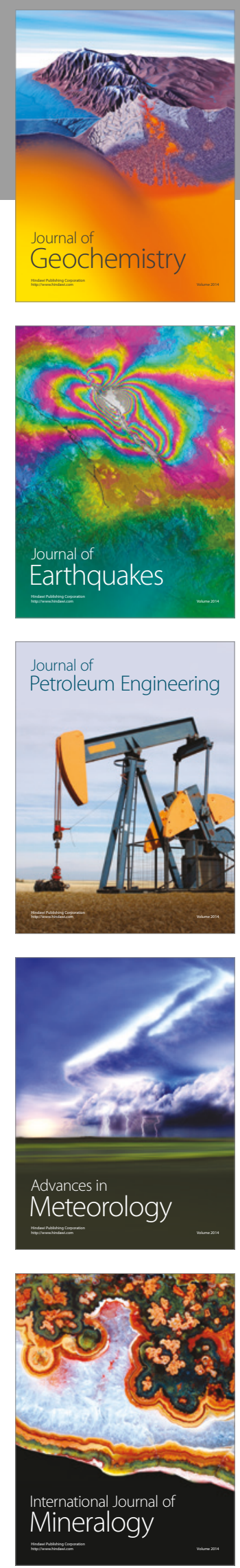
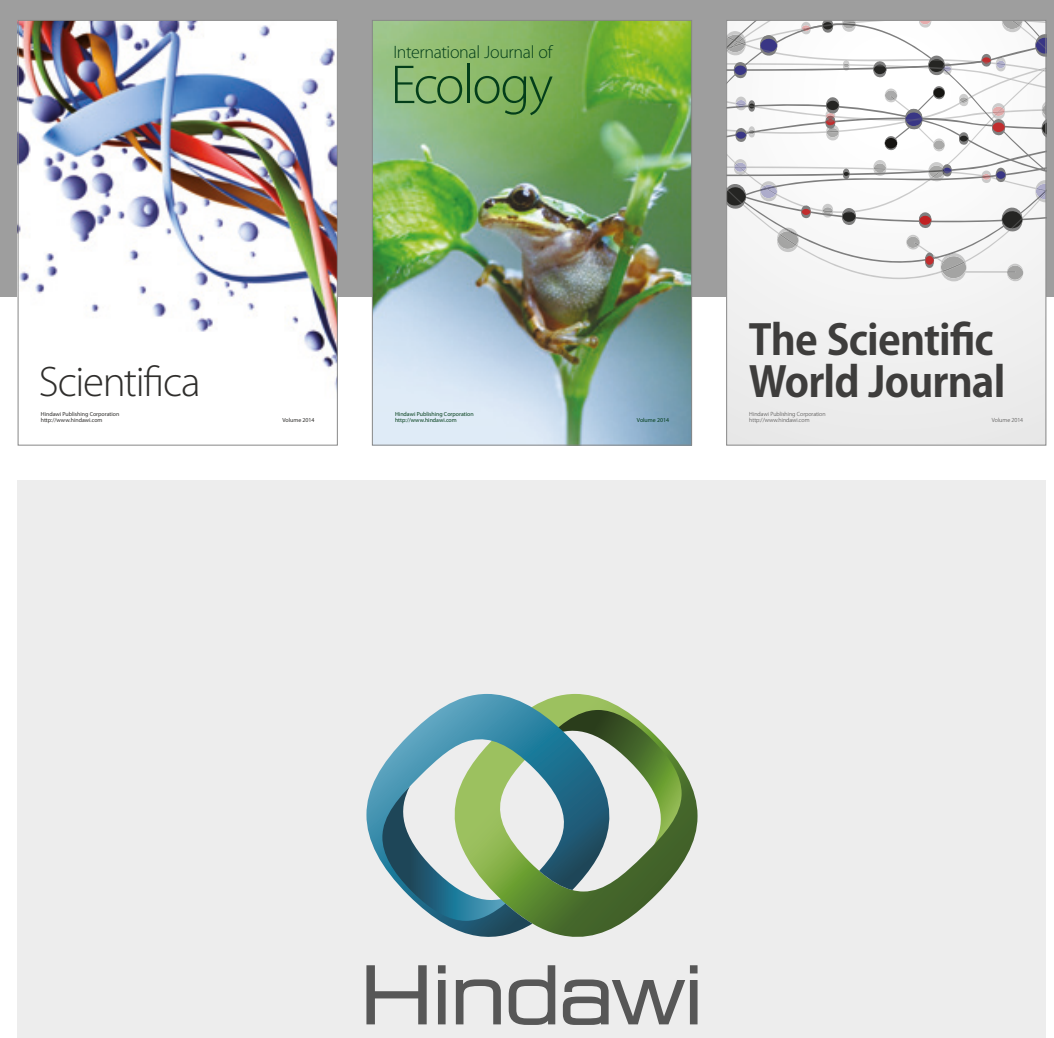

Submit your manuscripts at

https://www.hindawi.com
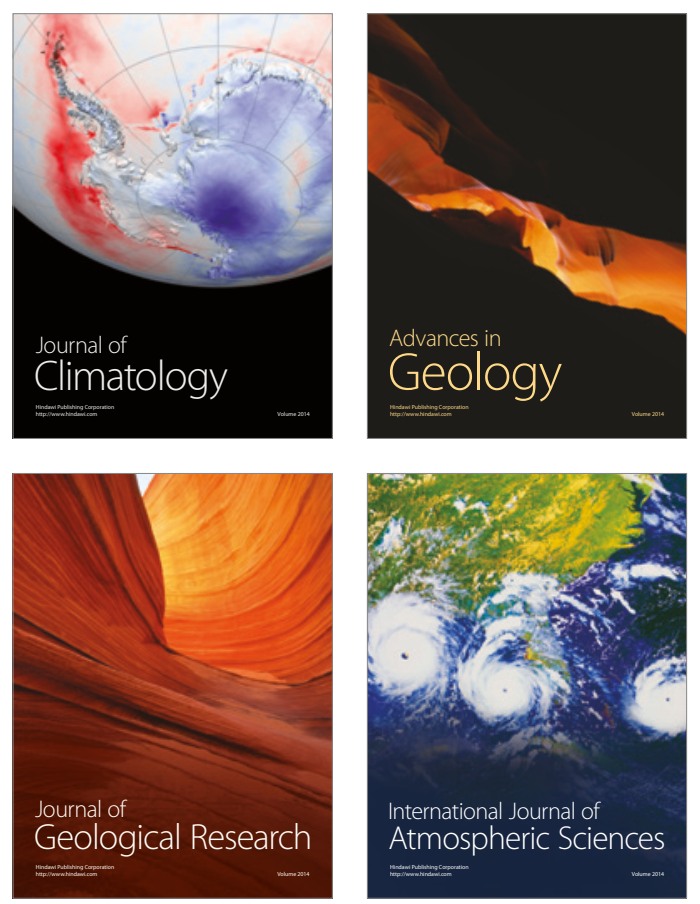

The Scientific

World Journal
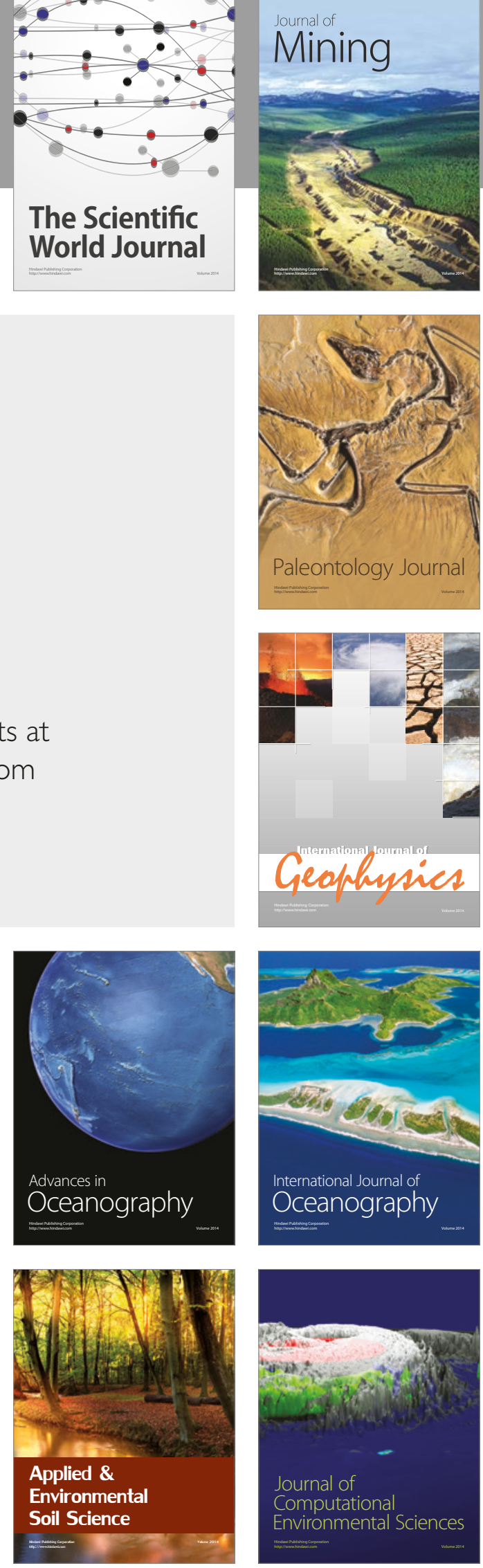